\title{
UNITARY EQUIVALENCE OF PROPER EXTENSIONS OF A SYMMETRIC OPERATOR AND THE WEYL FUNCTION
}

\author{
SEPPO HASSI, MARK MALAMUD, AND VADIM MOGILEVSKII
}

\begin{abstract}
Let $A$ be a densely defined simple symmetric operator in $\mathfrak{H}$, let $\Pi=$ $\left\{\mathcal{H}, \Gamma_{0}, \Gamma_{1}\right\}$ be a boundary triplet for $A^{*}$ and let $M(\cdot)$ be the corresponding Weyl function. It is known that the Weyl function $M(\cdot)$ determines the boundary triplet $\Pi$, in particular, the pair $\left\{A, A_{0}\right\}$, where $A_{0}:=A^{*}\left\lceil\operatorname{ker} \Gamma_{0}\left(=A_{0}^{*}\right)\right.$, uniquely up to unitary similarity. At the same time the Weyl function corresponding to a boundary triplet for a dual pair of operators defines it uniquely only up to weak similarity.

In this paper we consider symmetric dual pairs $\{A, A\}$ generated by $A \subset A^{*}$ and special boundary triplets $\widetilde{\Pi}$ for $\{A, A\}$. We are interested whether the result on unitary similarity remains valid provided that the Weyl function corresponding to $\widetilde{\Pi}$ is $\widetilde{M}(z)=$ $K^{*}(B-M(z))^{-1} K$, where $B$ is some non-self-adjoint bounded operator in $\mathcal{H}$. We specify some conditions in terms of the operators $A_{0}$ and $A_{B}=A^{*}\left\lceil\operatorname{ker}\left(\Gamma_{1}-B \Gamma_{0}\right)\right.$, which determine uniquely (up to unitary equivalence) the pair $\left\{A, A_{B}\right\}$ by the Weyl function $\widetilde{M}(\cdot)$. Moreover, it is shown that under some additional assumptions the Weyl function $M_{\Pi}(\cdot)$ of the boundary triplet $\Pi$ for the dual pair $\{A, A\}$ determines the triplet $\Pi$ uniquely up to unitary similarity. We obtain also some negative results demonstrating that in general the Weyl function $\widetilde{M}(\cdot)$ does not determine the operator $A_{B}$ even up to similarity.
\end{abstract}

\section{INTRODUCTION}

Let $\mathfrak{H}$ be a Hilbert space, let $A$ be a densely defined closed symmetric operator in $\mathfrak{H}$ with equal deficiency indices $n_{+}(A)=n_{-}(A) \leq \infty$, and let $A^{*}$ be the adjoint operator of $A$.

During the last three decades a new approach to the extension theory has been elaborated and has already attracted some attention. It is based on a concept of a boundary triplet $\Pi=\left\{\mathcal{H}, \Gamma_{0}, \Gamma_{1}\right\}$ for the operator $A^{*}$ (see [15]). The main ingredient of this approach is the following abstract Green's (Lagrange) identity

$$
\left(A^{*} f, g\right)-\left(f, A^{*} g\right)=\left(\Gamma_{1} f, \Gamma_{0} g\right)_{\mathcal{H}}-\left(\Gamma_{0} f, \Gamma_{1} g\right)_{\mathcal{H}}, \quad f, g \in \operatorname{dom} A^{*},
$$

where $\mathcal{H}$ is an auxiliary Hilbert space and $\Gamma_{0}, \Gamma_{1}$ are linear mappings from $A^{*}$ to $\mathcal{H}$ such that the mapping $\Gamma=\left(\begin{array}{ll}\Gamma_{0} & \Gamma_{1}\end{array}\right)^{\top}$ is surjective. A boundary triplet for $A^{*}$ always exists but is not unique. Its role in the extension theory is similar to that of a coordinate system in analytic geometry. It allows one to parameterize the set $\operatorname{Ext}_{A}$ of closed extensions $\widetilde{A}$ of $A$ satisfying $A \subset \widetilde{A} \subset A^{*}$ (proper extensions) in terms of abstract boundary conditions. Namely, the equality $\Theta=\Gamma \operatorname{dom} \widetilde{A}$ establishes a one-to-one correspondence between all extensions $A_{\Theta}:=\widetilde{A} \in \operatorname{Ext}_{A}$ and all closed linear relations $\Theta$ in $\mathcal{H}$. If $\Theta$ is the graph of a closed operator $B(\in \mathcal{C}(\mathcal{H})$ ), then the corresponding extension is given by

$$
\widetilde{A}=A_{B}=A^{*}\left[\operatorname{ker}\left(\Gamma_{1}-B \Gamma_{0}\right) .\right.
$$

1991 Mathematics Subject Classification. Primary 47A56, 47B25; Secondary 47A48, 47E05.

Key words and phrases. Symmetric operator, dual pair of operators, boundary triplet, Weyl function, unitary equivalence. 
The main analytical tool in this approach is the Weyl function $M(\cdot)$ corresponding to $\Pi$ that was introduced and investigated extensively in [12]. It is defined by

$$
\Gamma_{1} f_{z}=M(z) \Gamma_{0} f_{z}, \quad f_{z} \in \mathfrak{N}_{z}:=\operatorname{ker}\left(A^{*}-z\right), \quad z \in \mathbb{C} \backslash \mathbb{R} .
$$

It is shown in [12] that $M(\cdot)$ is well defined, takes values in $[\mathcal{H}]$ and is an $R[\mathcal{H}]$-function (Nevanlinna function), i.e., it is holomorphic in $\mathbb{C} \backslash \mathbb{R}, \operatorname{Im} z \operatorname{Im} M(z) \geq 0$ and $M^{*}(z)=$ $\overline{M(z)}, z \in \mathbb{C} \backslash \mathbb{R}$.

If the operator $A$ is simple, then the Weyl function $M(\cdot)$ determines the pair $\left\{A, A_{0}\right\}$, $A_{0}=A^{*}\left\lceil\operatorname{ker} \Gamma_{0}\right.$, as well as the boundary triplet $\Pi$ itself, uniquely up to unitary equivalence; see $[12,24]$. In particular, $M(\cdot)$ determines the extension $A_{B}$ given by (1.2) uniquely up to the unitary similarity. Note that the unique determination of the pair $\left\{A, A_{0}\right\}$ up to the unitary equivalence has been proved in [20,21] in terms of the so-called $Q$-functions.

The concept of a boundary triplet for $A^{*}$ has been extended to the case of dual pairs of closed operators $A, A^{T}$, i.e. pairs of operators satisfying $A \subset\left(A^{\top}\right)^{*}$; the definition reads as follows:

Definition 1.1. [23] Let $\left\{A, A^{T}\right\}$ be a dual pair of closed densely defined operators $A$ and $A^{T}$ in $\mathfrak{H}$. A collection $\Pi=\left\{\mathcal{H}_{0} \oplus \mathcal{H}_{1}, \Gamma, \Gamma^{\top}\right\}$, where $\mathcal{H}_{0}$ and $\mathcal{H}_{1}$ are Hilbert spaces and

$$
\Gamma=\left(\begin{array}{c}
\Gamma_{0} \\
\Gamma_{1}
\end{array}\right): \operatorname{dom}\left(A^{\top}\right)^{*} \rightarrow \mathcal{H}_{0} \oplus \mathcal{H}_{1}, \quad \Gamma^{\top}=\left(\begin{array}{l}
\Gamma_{0}^{\top} \\
\Gamma_{1}^{\top}
\end{array}\right): \operatorname{dom} A^{*} \rightarrow \mathcal{H}_{1} \oplus \mathcal{H}_{0}
$$

are linear mappings, is called a boundary triplet for $\left\{A, A^{\top}\right\}$ if the mappings $\Gamma$ and $\Gamma^{\top}$ are surjective and for every $f \in \operatorname{dom}\left(A^{\top}\right)^{*}$ and $g \in \operatorname{dom} A^{*}$ the following abstract Green's identity holds

$$
\left(\left(A^{\top}\right)^{*} f, g\right)-\left(f, A^{*} g\right)=\left(\Gamma_{1} f, \Gamma_{0}^{\top} g\right)-\left(\Gamma_{0} f, \Gamma_{1}^{\top} g\right) .
$$

First constructions of boundary triplets for a dual pair of non-self-adjoint elliptic operators as well as their applications to non-local boundary value problems for elliptic operators in domains with smooth boundary go back to the classical papers by M.I. Visik [31] and G. Grubb [16].

In $[27,29]$ a concept of the Weyl function $M_{\Pi}(\cdot)$ corresponding to the triplet $\Pi$ have also been extended to the case of dual pairs $\left\{A, A^{T}\right\}$. The considerations in $[27,29]$ have been inspired by investigations of V.B. Lidskii [22] in the spectral theory of the SturmLiouville operator $-\frac{d^{2}}{d x^{2}}+q$ with complex-valued potential; there the Weyl function was introduced by extending the Weyl limit-circle procedure. It turned out that the abstract Weyl function $M_{\Pi}(\cdot)$ from $[27,29]$ coincides with that from [22]. Moreover, it was shown in [29] that for each boundary triplet $\Pi$ for $\left\{A, A^{\top}\right\}$ the extension $A_{0}:=\left(A^{\top}\right)^{*}\left\lceil\operatorname{ker} \Gamma_{0}\right.$ and the Weyl function $M_{\Pi}(\cdot)$ coincide, respectively, with the main operator and the transfer function of some linear stationary system (in the sense of $[3,4]$ ). Further investigations in this topic have been motivated by possible applications to boundary value problems for non-self-adjoint differentials operators (see $[29,9,10,11,17,25]$ and references therein).

It is shown in [28] that in the case of a simple dual pair $\left\{A, A^{\top}\right\}$ the Weyl function $M_{\Pi}(\cdot)$ determines the boundary triplet $\Pi$ as well as the proper extension $A_{B}$ uniquely up to weak similarity (similar result for linear stationary systems was earlier obtained in [3]). Note that weak similarity does not preserve the spectral properties and even the spectrum of the main operator. In recent publications [5] and $[1,2]$ it was shown that in two special cases the Weyl function determines the operator $A_{B}$ up to similarity [5] and up to unitary similarity $[1,2]$, respectively.

In this paper we consider only special dual pairs $\{A, A\}$ generated by a symmetric, not necessarily densely defined, operator $A$. Moreover, we consider only special boundary 
triplets $\Pi=\left\{\mathcal{H} \oplus \mathcal{H}, \Gamma, \Gamma^{\top}\right\}$ for the dual pair $\{A, A\}$ such that the corresponding Weyl function $M_{\Pi}(\cdot)$ is

$$
\widetilde{M}(z):=M_{\Pi}(z)=K^{*}(B-M(z))^{-1} K, \quad z \in \rho\left(A_{B}\right),
$$

where $M(\cdot)$ is the Weyl function of $A$ defined by (1.3) and $B$ is a bounded non-selfadjoint $\left(B \neq B^{*}\right)$ operator in $\mathcal{H}$. The latter means, in particular, that $A_{B}$ is the almost solvable extension of $A$ in the sense of [13].

The Weyl function $M_{\Pi}(\cdot)$ of the form (1.4) is no longer Nevanlinna function in $\mathbb{C}_{ \pm}$. However, if $B$ is accumulative, then the Weyl function $M_{\Pi}(\cdot)$ of the form (1.4) is a Nevanlinna function in $\mathbb{C}_{+}$, i.e. it is holomorphic in $\mathbb{C}_{+}$and $\operatorname{Im} M_{\Pi}(z) \geq 0, z \in \mathbb{C}_{+}$. It follows that $M_{\Pi}(\cdot)$ admits a classical integral representation (cf. (2.3)) only in $\mathbb{C}_{+}$.

We are interested in sufficient (and necessary) conditions in terms of the operators $A_{B}$ and $A_{0}=A_{0}^{*}$ that make it possible to determine the pair $\left\{A, A_{B}\right\}$ uniquely (up to the unitary equivalence) by the Weyl function (1.4). More precisely, we consider the following problem:

Given a simple symmetric operator $A^{(j)}$, a boundary triplet $\Pi_{j}=\left\{\mathcal{H}, \Gamma_{0}^{(j)}, \Gamma_{1}^{(j)}\right\}$ for $A^{(j) *}$, the corresponding Weyl function $M_{j}(\cdot)$, a proper extension $A_{B_{j}}^{(j)}=A^{(j) *}\left\lceil\operatorname{ker}\left(\Gamma_{1}^{(j)}-\right.\right.$ $\left.B_{j} \Gamma_{0}^{(j)}\right), j \in\{1,2\}$, and a domain $\Omega \subset \rho\left(A_{B_{1}}^{(1)}\right) \cap \rho\left(A_{B_{2}}^{(2)}\right) \subset \mathbb{C}$. When the equality

$$
\widetilde{M}_{1}(z)=K_{1}^{*}\left(B_{1}-M_{1}(z)\right)^{-1} K_{1}=K_{2}^{*}\left(B_{2}-M_{2}(z)\right)^{-1} K_{2}=\widetilde{M}_{2}(z), \quad z \in \Omega,
$$

yields the unitary similarity of the pairs of operators $\left\{A_{B_{1}}^{(1)}, A_{0}^{(1)}\right\}$ and $\left\{A_{B_{2}}^{(2)}, A_{0}^{(2)}\right\} ?$

We show (cf. Theorem 3.9) that the answer is positive at least in the following two cases:

(i) $\Omega \cap \mathbb{C}_{ \pm} \neq \emptyset$;

(ii) $\Omega \subset \mathbb{C}_{+}$and the ac-part $E_{j}^{a c}(\cdot)$ of the spectral measure $E_{j}(\cdot)$ of $A_{0}^{(j)}, j \in\{1,2\}$, is not equivalent to the Lebesgue measure.

In particular, both assumptions (i) and (ii) are satisfied provided that $\Omega \cap \mathbb{R} \neq \emptyset$. It is emphasized however, that condition (ii) is not necessary for the unitary equivalence of $A_{B_{1}}$ and $A_{B_{2}}$. Moreover, the unitary equivalence might happen even if both $E_{1}^{a c}(\cdot)$ and $E_{2}^{a c}(\cdot)$ are spectrally equivalent to the Lebesgue measure (see Remark 3.3).

To include in our considerations bounded operators $A_{0}=A_{0}^{*}$ we consider dual pairs $\{A, A\}$ with a bounded nondensely defined symmetric operator $A$. We show that the Weyl function $M_{\Pi}(\cdot)$ corresponding to a special boundary triplet $\Pi=\left\{\mathcal{H} \oplus \mathcal{H}, \Gamma, \Gamma^{\top}\right\}$ for $\{A, A\}$ is

$$
M_{\Pi}(z)=\mathcal{F}+K^{*}\left(A_{0}-z\right)^{-1} K, \quad z \in \rho\left(A_{0}\right),
$$

(c.f. (1.4)), where $A_{0}=\operatorname{ker} \Gamma_{0} \in[\mathfrak{H}]$ and $K \in[\mathcal{H}, \mathfrak{H}], \mathcal{F} \in[\mathcal{H}]$ are the operators defined in terms of the boundary triplet $\Pi$. Since $A_{0}$ is bounded, $M_{\Pi}(\infty)=\mathcal{F}$ and it follows from each of the assumptions (i) and (ii) that $M_{\Pi}(\cdot)$ determines the pair $\left\{A_{0}, A_{B}\right\}$ uniquely up to the unitary equivalence. In fact, in this case a stronger statement is valid: the Weyl function $M_{\Pi}(\cdot)$ determines the boundary triplet $\Pi$ uniquely up to unitary equivalence (see Theorem 4.6). A similar result is also valid for unbounded $A$ provided that $\rho\left(A_{0}\right) \cap \mathbb{R} \neq \emptyset$ (cf. Theorem 4.7). This result can be reformulated as follows: the transfer function of a special linear stationary system determines it uniquely up to unitary similarity (see Remark 4.9 for details).

In the last section we present some negative results demonstrating that in general the function $\widetilde{M}(\cdot)$ of the form (1.4) does not determine the operator $A_{B}$ uniquely even up to the similarity. For instance, it is shown that for any symmetric operator $A^{(1)}$, 
any Weyl function $M_{1}(\cdot)$ of $A^{(1)}$, and any accumulative $B_{1} \in[\mathcal{H}]$ there exists a (nonunique) simple symmetric operator $A^{(2)}$ and a (non-unique) dissipative operator $B_{2} \in[\mathcal{H}]$ such that equality (1.5) holds with $K_{1}=K_{2}=I_{\mathcal{H}}$ and $\Omega \subset \mathbb{C}_{+}$. At the same time the operators $A_{B_{1}}$ and $A_{B_{2}}$ have different spectra, hence cannot be similar. Observe also that without additional restrictions the Weyl function $\widetilde{M}(\cdot)$ does not determine the extension $A_{B}$ uniquely up to the unitary similarity even in the case of the accumulative $B$ (see Remark 5.2(4)).

Finally, we present some explicit examples illustrating the above effect.

The main results of the paper have been announced without proofs in [19].

Notation. Throughout the paper $\mathfrak{H}$ and $\mathcal{H}$ are assumed to be separable Hilbert spaces, the set of bounded linear operators from $\mathcal{H}_{0}$ to $\mathcal{H}_{1}$ is denoted by $\left[\mathcal{H}_{0}, \mathcal{H}_{1}\right]$, we also write $[\mathcal{H}]:=[\mathcal{H}, \mathcal{H}]$. Further, $P_{\mathcal{L}} \in[\mathfrak{H}]$ denotes the orthoprojector in $\mathfrak{H}$ onto the subspace $\mathcal{L} \subset \mathfrak{H}$. The open upper and lower half-plane of $\mathbb{C}$ are denoted by $\mathbb{C}_{+}$and $\mathbb{C}_{-}$, respectively.

\section{Preliminaries}

2.1. Linear relations. Let $\mathcal{H}_{0}$ and $\mathcal{H}_{1}$ be Hilbert spaces. A linear relation $T$ from $\mathcal{H}_{0}$ to $\mathcal{H}_{1}$ is a linear manifold in $\mathcal{H}_{0} \oplus \mathcal{H}_{1}$. In particular, the set of all closed linear relations from $\mathcal{H}_{0}$ to $\mathcal{H}_{1}$ is denoted by $\widetilde{\mathcal{C}}\left(\mathcal{H}_{0}, \mathcal{H}_{1}\right)$, and $\widetilde{\mathcal{C}}(\mathcal{H}):=\widetilde{\mathcal{C}}(\mathcal{H}, \mathcal{H})$. For each $T \in \widetilde{\mathcal{C}}\left(\mathcal{H}_{0}, \mathcal{H}_{1}\right)$ the domain, the range, the kernel and the multi-valued part of $T$ are denoted by $\operatorname{dom} T, \operatorname{ran} T, \operatorname{ker} T$, and mul $T, \operatorname{respectively.~The~notations~} \overline{\operatorname{dom}} T$ and $\overline{\operatorname{ran}} T$ stand for the closures of the domain $\operatorname{dom} T$ and the range $\operatorname{ran} T$ of $T$. Systematically a closed linear operator $T$ from $\mathcal{H}_{0}$ to $\mathcal{H}_{1}$ will be identified with its graph

$$
\operatorname{gr} T=\{\{f, T f\}: f \in \operatorname{dom} T\} \in \widetilde{\mathcal{C}}\left(\mathcal{H}_{0}, \mathcal{H}_{1}\right) .
$$

If $T \in \widetilde{\mathcal{C}}\left(\mathcal{H}_{0}, \mathcal{H}_{1}\right)$ then the inverse linear relation $T^{-1}$ is given by

$$
T^{-1}=\left\{\left\{f^{\prime}, f\right\}:\left\{f, f^{\prime}\right\} \in T\right\}\left(\in \widetilde{\mathcal{C}}\left(\mathcal{H}_{1}, \mathcal{H}_{0}\right)\right) .
$$

Furthermore, the adjoint linear relation $T^{*}$ is defined as

$$
T^{*}=\left\{\left\{g, g^{\prime}\right\} \in \mathcal{H}_{1} \oplus \mathcal{H}_{0}:\left(f^{\prime}, g\right)=\left(f, g^{\prime}\right), \quad\left\{f, f^{\prime}\right\} \in T\right\} \in \widetilde{\mathcal{C}}\left(\mathcal{H}_{1}, \mathcal{H}_{0}\right) .
$$

For a linear relation $T \in \widetilde{\mathcal{C}}\left(\mathcal{H}_{0}, \mathcal{H}_{1}\right)$ the following notations are frequently used:

$0 \in \rho(T)$ if $\operatorname{ker} T=\{0\}$ and $\operatorname{ran} T=\mathcal{H}_{1}$, or equivalently, if $T^{-1} \in\left[\mathcal{H}_{1}, \mathcal{H}_{0}\right]$;

$0 \in \widehat{\rho}(T)$ if $\operatorname{ker} T=\{0\}$ and $\overline{\operatorname{ran}} T=\operatorname{ran} T \neq \mathcal{H}_{1}$;

$0 \in \sigma_{c}(T)$ if $\operatorname{ker} T=\{0\}$ and $\overline{\operatorname{ran}} T=\mathcal{H}_{1} \neq \operatorname{ran} T$;

$0 \in \sigma_{p}(T)$ if $\operatorname{ker} T \neq\{0\}$;

$0 \in \sigma_{r}(T)$ if $0 \in \sigma(T) \backslash\left(\sigma_{p}(T) \cup \sigma_{c}(T)\right)$.

For any $T \in \widetilde{\mathcal{C}}(\mathcal{H})$ denote by $\rho(T)=\{\lambda \in \mathbb{C}: 0 \in \rho(T-\lambda)\}$ and $\widehat{\rho}(T)=\{\lambda \in \mathbb{C}$ : $0 \in \widehat{\rho}(T-\lambda)\}$ the resolvent set and the set of regular type points of $T$, respectively. The spectrum of $T$ is given by $\sigma(T)=\mathbb{C} \backslash \rho(T)$. It admits the following classification:

$\sigma_{c}(T)=\left\{\lambda \in \mathbb{C}: 0 \in \sigma_{c}(T-\lambda)\right\}$ is the continuous spectrum;

$\sigma_{p}(T)=\left\{\lambda \in \mathbb{C}: 0 \in \sigma_{p}(T-\lambda)\right\}$ is the point spectrum;

$\sigma_{r}(T)=\left\{\lambda \in \mathbb{C}: 0 \in \sigma_{r}(T-\lambda)\right\}$ is the residual spectrum.

A linear relation $T \in \widetilde{\mathcal{C}}(\mathcal{H})$ is called symmetric if $T \subset T^{*}$ and self-adjoint if $T=T^{*}$.

2.2. Operator measures. Here some known facts on operator measures are recalled. Let $\mathcal{H}$ be a separable Hilbert space, let $\mathcal{B}(\mathbb{R})$ be the Borel $\sigma$-algebra of the real line $\mathbb{R}$ and let $\mathcal{B}_{b}(\mathbb{R})$ be the ring of all bounded sets in $\mathcal{B}(\mathbb{R})$.

Definition 2.1. (i) A mapping $\Sigma(\cdot): \mathcal{B}_{b}(\mathbb{R}) \rightarrow[\mathcal{H}]$ is called an operator measure if $\Sigma(\emptyset)=0, \Sigma(\delta)=\Sigma(\delta)^{*} \geq 0\left(\delta \in \mathcal{B}_{b}(\mathbb{R})\right)$, and the function $\Sigma(\cdot)$ is strongly countably additive. 
(ii) An operator measure $\Sigma(\cdot)$ is called bounded if it is defined on $\mathcal{B}(\mathbb{R})$.

(iii) A bounded operator measure $\Sigma(\cdot)=: E(\cdot)$ is said to be orthogonal if $E(\mathbb{R})=I$ and $E^{2}(\delta)=E(\delta)$ (i.e., $E(\delta)$ is the orthoprojector in $\mathcal{H}$ ) .

By the spectral theorem [7], there exists a one-to-one correspondence between the orthogonal measures $E(\cdot)$ in $\mathcal{H}$ and self-adjoint operators $T=T^{*}$ in $\mathcal{H}$. It is given by the decomposition

$$
T=\int_{\mathbb{R}} t d E(t), \quad \operatorname{dom} T=\left\{f \in \mathcal{H}: \int_{\mathbb{R}} t^{2} d(E(t) f, f)<\infty\right\} .
$$

The measure $E(\cdot)$ is called the spectral measure of $T$.

The operator measure $\Sigma_{1}$ is called absolutely continuous with respect to the measure $\Sigma_{2}$ (in symbols: $\Sigma_{1} \prec \Sigma_{2}$ ) if $\Sigma_{2}(\delta)=0$ implies $\Sigma_{1}(\delta)=0$ for $\delta \in \mathcal{B}_{b}(\mathbb{R})$. The measures $\Sigma_{1}$ and $\Sigma_{2}$ are called equivalent $\left(\Sigma_{1} \sim \Sigma_{2}\right)$ if $\Sigma_{1} \prec \Sigma_{2}$ and $\Sigma_{2} \prec \Sigma_{1}$.

In the sequel we denote by $m(\cdot)$ the (scalar) Lebesgue measure in $\mathbb{R}$. The operator measure $\Sigma(\cdot)$ is called singular (with respect to $m(\cdot)$ ) if there exists a set $\delta_{0} \in \mathcal{B}(\mathbb{R}$ ) such that $m\left(\delta_{0}\right)=0$ and $\Sigma(\delta)=\Sigma\left(\delta \cap \delta_{0}\right)$ for all $\delta \in \mathcal{B}_{b}(\mathbb{R})$. The singularity of $\Sigma(\cdot)$ is denoted by $\Sigma \perp m$.

Each operator measure $\Sigma(\cdot)$ admits the Lebesgue decomposition

$$
\Sigma=\Sigma^{a c}+\Sigma^{s}, \quad \text { where } \quad \Sigma^{a c} \prec m \text { and } \quad \Sigma^{s} \perp m .
$$

The operator measures $\Sigma^{a c}$ and $\Sigma^{s}$ are called the absolutely continuous and singular parts of $\Sigma$, respectively. If the measure $\Sigma(\cdot)=E(\cdot)$ is orthogonal, then its absolutely continuous and singular parts $E^{a c}(\cdot)$ and $E^{s}(\cdot)$ are also orthogonal, $E^{a c}(\cdot) E^{s}(\cdot)=0$, and the decomposition in (2.2) can be rewritten as $E=E^{a c} \oplus E^{s}$.

For a self-adjoint operator $T$ in $\mathcal{H}$ with the spectral measure $E(\cdot)$ denote $\mathcal{H}_{\tau}:=E^{\tau} \mathcal{H}$ and $T_{\tau}=T\left\lceil\mathcal{H}_{\tau}, \tau=a c, s\right.$. This yields the decompositions $\mathcal{H}=\mathcal{H}_{a c} \oplus \mathcal{H}_{s}$ and $T=$ $T_{a c} \oplus T_{s}$. The operators $T_{a c}$ and $T_{s}$ are called the $a c$-part and the singular part of $T$, respectively. The spectrum $\sigma\left(T_{a c}\right)\left(\sigma\left(T_{s}\right)\right)$ is called the $a c$-spectrum (resp. the singular spectrum) of $T$.

Recall that each self-adjoint relation $T \in \widetilde{\mathcal{C}}(\mathcal{H})$ admits the decomposition

$$
T=T^{\prime} \oplus \widehat{\operatorname{mul}} T, \quad \widehat{\operatorname{mul}} T=\{0\} \oplus \operatorname{mul} T,
$$

where $T^{\prime}$ is a self-adjoint operator (the operator part of $T$ ) in $\mathcal{H}^{\prime}:=\mathcal{H} \ominus \operatorname{mul} T$.

Definition 2.2. Let $T=T^{*} \in \widetilde{\mathcal{C}}(\mathcal{H})$ and mul $T \neq \mathcal{H}$, i.e., $\left.\mathcal{H}^{\prime} \neq\{0\}\right)$. Then the spectral measure of $T$ is defined to be the spectral measure $E(\cdot): \mathcal{B}(\mathbb{R}) \rightarrow\left[\mathcal{H}^{\prime}\right]$ of its operator part $T^{\prime}=\left(T^{\prime}\right)^{*}$. Moreover, the ac-spectrum $\sigma_{a c}(T)$ and singular spectrum $\sigma_{s}(T)$ of $T$ are defined as $\sigma_{a c}(T):=\sigma_{a c}\left(T^{\prime}\right)$ and $\sigma_{s}(T):=\sigma_{s}\left(T^{\prime}\right)$, respectively.

2.3. $R$-Functions. A holomorphic operator-valued function $F(\cdot): \mathbb{C} \backslash \mathbb{R} \rightarrow[\mathcal{H}]$ is called an $R$-function (Nevanlinna function) if $\operatorname{Im} z \cdot \operatorname{Im} F(z) \geq 0$ and $F^{*}(z)=F(\bar{z}), z \in \mathbb{C} \backslash \mathbb{R}$. The class of $R$-functions with values in $[\mathcal{H}]$ is denoted by $R[\mathcal{H}]$. Every $F(\cdot) \in R[\mathcal{H}]$ admits an integral representation of the form (see [8])

$$
F(z)=C+D z+\int_{\mathbb{R}}\left(\frac{1}{t-z}-\frac{t}{1+t^{2}}\right) d \Sigma, \quad z \in \mathbb{C} \backslash \mathbb{R},
$$

where $C, D \in[\mathcal{H}], C=C^{*}, D \geq 0$ and $\Sigma(\cdot): \mathcal{B}_{b}(\mathbb{R}) \rightarrow[\mathcal{H}]$ is an operator measure satisfying

$$
\int_{\mathbb{R}} \frac{d \Sigma}{1+t^{2}} \in[\mathcal{H}]
$$

(the integrals are understood in the strong sense). The operator measure $\Sigma(\cdot)$ and the operators $C, D$ in $(2.3)$ are called the spectral measure and parameters of $F(\cdot)$, 
respectively. They are uniquely defined by $F(\cdot)$. Moreover, a distribution operator function $\Sigma(\cdot): \mathbb{R} \rightarrow[\mathcal{H}]$ defined by

$$
\Sigma(t)=\left\{\begin{array}{cc}
\Sigma([0, t)), & t>0 \\
0, & t=0 \\
-\Sigma([t, 0)), & t<0
\end{array}\right.
$$

is called the (normalized) spectral function of $F(\cdot)$.

2.4. Boundary triplets and the Weyl functions. Let $\mathfrak{H}$ be a Hilbert space, let $A$ be a closed symmetric operator in $\mathfrak{H}$, not necessarily densely defined, and let $A^{*}(\in \widetilde{\mathcal{C}}(\mathfrak{H}))$ be the adjoint of $A$. Assume also that the operator $A$ has equal deficiency indices $n_{ \pm}(A)=\operatorname{dim}\left(\operatorname{ker}\left(A^{*} \mp i\right)\right) \leq \infty$.

Here we briefly recall the basic facts on boundary triplets and the corresponding Weyl functions following $[15,12,24,14]$.

Definition $2.3([15,24])$. A triplet $\Pi=\left\{\mathcal{H}, \Gamma_{0}, \Gamma_{1}\right\}$, where $\mathcal{H}$ is a Hilbert space and $\Gamma_{0}, \Gamma_{1}: A^{*} \rightarrow \mathcal{H}$ are linear mappings, is called a boundary triplet for $A^{*}$ if the following "abstract Green's identity" holds

$$
\left(f^{\prime}, g\right)-\left(f, g^{\prime}\right)=\left(\Gamma_{1} \widehat{f}, \Gamma_{0} \widehat{g}\right)_{\mathcal{H}}-\left(\Gamma_{0} \widehat{f}, \Gamma_{1} \widehat{g}\right)_{\mathcal{H}}, \quad \widehat{f}=\left\{f, f^{\prime}\right\}, \widehat{g}=\left\{g, g^{\prime}\right\} \in A^{*}
$$

and the mapping $\Gamma:=\left(\begin{array}{ll}\Gamma_{0} & \Gamma_{1}\end{array}\right)^{\top}: A^{*} \rightarrow \mathcal{H} \oplus \mathcal{H}$ is surjective.

Note that $A^{*}$ is a densely defined operator if and only if $\operatorname{dom} A$ is dense in $\mathfrak{H}$. In this case the identity $(2.6)$ can be rewritten in the equivalent form

$$
\left(A^{*} f, g\right)-\left(f, A^{*} g\right)=\left(\Gamma_{1} f, \Gamma_{0} g\right)_{\mathcal{H}}-\left(\Gamma_{0} f, \Gamma_{1} g\right)_{\mathcal{H}}, \quad f, g \in \operatorname{dom} A^{*} .
$$

A boundary triplet $\Pi=\left\{\mathcal{H}, \Gamma_{0}, \Gamma_{1}\right\}$ for $A^{*}$ exists whenever $n_{+}(A)=n_{-}(A)$. Moreover, one has $n_{ \pm}(A)=\operatorname{dim} \mathcal{H}, \operatorname{ker} \Gamma_{j}(j=1,2)$ is selfadjoint, and $\operatorname{ker} \Gamma_{0} \cap \operatorname{ker} \Gamma_{1}=A$. It is known that $\Gamma_{0}, \Gamma_{1} \in\left[A^{*}, \mathcal{H}\right]$, that is the operators $\Gamma_{0}$ and $\Gamma_{1}$ are bounded with respect to the graph topology on $A^{*}$.

Definition 2.4. A closed linear relation $\widetilde{A} \in \widetilde{\mathcal{C}}(\mathfrak{H})$ is called a proper extension of $A$, if $A \varsubsetneqq \widetilde{A} \varsubsetneqq A^{*}$. The set of proper extensions augmented by $A$ and $A^{*}$ is denoted by $\operatorname{Ext}_{A}$.

Clearly, every self-adjoint extension $\widetilde{A}=\widetilde{A}^{*}$ is automatically proper, i.e., $\widetilde{A} \in \operatorname{Ext}_{A}$. Moreover, every closed dissipative extension $\widetilde{A}$ is also proper (see [24]). With a boundary triplet $\Pi$ one typically fixes two extensions $A_{j}:=\operatorname{ker} \Gamma_{j}, j \in\{0,1\}$ of $A$, which are selfadjoint in view of Proposition 2.5 below. Conversely, for every $A_{0}=A_{0}^{*} \in \operatorname{Ext}_{A}$ there exists a (non-unique) boundary triplet $\Pi=\left\{\mathcal{H}, \Gamma_{0}, \Gamma_{1}\right\}$ for $A^{*}$ such that $A_{0}=\operatorname{ker} \Gamma_{0}$.

Using the concept of a boundary triplet one can parameterize the set of all proper extensions of $A$ by means of the set $\widetilde{\mathcal{C}}(\mathcal{H})$ of closed linear relations in $\mathcal{H}$.

Proposition 2.5. Let $\Pi=\left\{\mathcal{H}, \Gamma_{0}, \Gamma_{1}\right\}$ be a boundary triplet for $A^{*}$. Then the mapping

$$
\operatorname{Ext}_{A} \ni \widetilde{A} \rightarrow \Gamma \widetilde{A}=\left\{\left\{\Gamma_{0} \widehat{f}, \Gamma_{1} \widehat{f}\right\}: \widehat{f} \in \widetilde{A}\right\}=: \Theta \in \widetilde{\mathcal{C}}(\mathcal{H})
$$

establishes a bijective correspondence between the sets $\operatorname{Ext}_{A}$ and $\widetilde{\mathcal{C}}(\mathcal{H})$. We put $A_{\Theta}:=\widetilde{A}$ where $\Theta$ is defined by (2.8). Moreover, the following statements hold:

(i) if $\Theta:=B \in \mathcal{C}(\mathcal{H})$ is an operator, then (2.8) takes the form

$$
A_{B}=\operatorname{ker}\left(\Gamma_{1}-B \Gamma_{0}\right) ;
$$

(ii) the extension $A_{\Theta} \in \operatorname{Ext}_{A}$ is $m$-dissipative, $m$-accumulative, self-adjoint, if and only if so is the corresponding linear (boundary) relation $\Theta \in \widetilde{\mathcal{C}}(\mathcal{H})$. 
The linear relation $\Theta$ (the operator $B$ ) is called the boundary relation (the boundary operator) or the coordinate of the extension $A_{\Theta}$. In particular, $A_{j}:=\operatorname{ker} \Gamma_{j}=A_{\Theta_{j}}, j \in$ $\{0,1\}$, where $\Theta_{0}:=\{0\} \times \mathcal{H}$ and $\Theta_{1}:=\mathcal{H} \times\{0\}$. Hence $A_{j}=A_{j}^{*}$ since clearly $\Theta_{j}=\Theta_{j}^{*}$. In the sequel the extension $A_{0}$ is usually regarded as a reference self-adjoint extension.

It is well known that Weyl functions play an important role in the direct and inverse spectral theory of singular Sturm-Liouville operators. In the papers $[12,24]$ the concept of Weyl function was generalized to the case of an arbitrary symmetric operator $A$ with $n_{+}(A)=n_{-}(A)$. Some basic facts on the Weyl functions and $\gamma$-fields are now briefly recalled.

Let $\mathfrak{N}_{z}(A):=\operatorname{ker}\left(A^{*}-z\right)$ be the defect subspace of $A$ and let $\widehat{\mathfrak{N}}_{z}(A):=\left\{\left\{f_{z}, z f_{z}\right\}\right.$ : $\left.f_{z} \in \mathfrak{N}_{z}(A)\right\}$. Clearly, $\widehat{\mathfrak{N}}_{z}(A)$ is a (closed) subspace in $A^{*}$. Denote by $\pi_{1}$ the orthoprojector in $\mathfrak{H} \oplus \mathfrak{H}$ onto $\mathfrak{H} \oplus\{0\}$.

Definition 2.6 $([12,24])$. Let $\Pi=\left\{\mathcal{H}, \Gamma_{0}, \Gamma_{1}\right\}$ be a boundary triplet for $A^{*}$. The operator functions $\gamma(\cdot): \rho\left(A_{0}\right) \rightarrow[\mathcal{H}, \mathfrak{H}]$ and $M(\cdot): \rho\left(A_{0}\right) \rightarrow[\mathcal{H}]$ defined by

$$
\gamma(z):=\pi_{1}\left(\Gamma_{0} \uparrow \widehat{\mathfrak{N}}_{z}(A)\right)^{-1}, \quad \Gamma_{1} \uparrow \widehat{\mathfrak{N}}_{z}(A)=M(z) \Gamma_{0} \uparrow \widehat{\mathfrak{N}}_{z}(A), \quad z \in \rho\left(A_{0}\right),
$$

are called the $\gamma$-field and the Weyl function, respectively, corresponding to $\Pi$.

It is shown in $[12,24]$ that the operator functions $\gamma(\cdot)$ and $M(\cdot)$ are well defined and holomorphic on $\rho\left(A_{0}\right)$. Moreover, $M(\cdot) \in R^{u}[\mathcal{H}]$, i.e., $M(\cdot) \in R[\mathcal{H}]$ and $0 \in \rho(\operatorname{Im}(M(i)))$. A symmetric operator $A$ in $\mathfrak{H}$ is called simple if there is no nontrivial decomposition

$$
\mathfrak{H}=\mathfrak{H}_{1} \oplus \mathfrak{H}_{2}, \quad A=A_{1} \oplus A_{2},
$$

where $\mathfrak{H}_{1} \neq\{0\}, A_{1}=A_{1}^{*} \in \mathcal{C}\left(\mathfrak{H}_{1}\right)$ and $A_{2}$ is a symmetric operator in $\mathfrak{H}_{2}$.

Proposition 2.7. $[24,14,6]$ Let $A$ be a simple symmetric operator in $\mathfrak{H}$ and let $\Pi=$ $\left\{\mathcal{H}, \Gamma_{0}, \Gamma_{1}\right\}$ be a boundary triplet for $A^{*}$ with $A_{0}=\operatorname{ker} \Gamma_{0}$. Let $M(\cdot)$ be the corresponding Weyl function and let $\Sigma(\cdot)$ be the spectral measure of $M(\cdot)$. If mul $A_{0} \neq \mathfrak{H}$, then the spectral measure $E(\cdot)$ of $A_{0}$ and the measure $\Sigma(\cdot)$ are equivalent, $E \sim \Sigma$. In particular, $E^{a c} \sim \Sigma^{a c}$ and $E^{s} \sim \Sigma^{s}$.

A description of spectra of proper extensions $A_{\Theta}$ of $A$ in terms of $\Theta$ and the corresponding Weyl function $M(\cdot)$ is given as follows.

Proposition 2.8. $[12,14]$ Let $\Pi=\left\{\mathcal{H}, \Gamma_{0}, \Gamma_{1}\right\}$ be a boundary triplet for $A^{*}$ with the Weyl function $M(\cdot)$ and let $\Theta \in \widetilde{\mathcal{C}}(\mathcal{H})$. Then for all $\lambda \in \rho\left(A_{0}\right)$ the following equivalences hold:

$$
\begin{gathered}
\lambda \in \rho\left(A_{\Theta}\right) \Longleftrightarrow 0 \in \rho(\Theta-M(\lambda)), \\
\lambda \in \sigma_{j}\left(A_{\Theta}\right) \Longleftrightarrow 0 \in \sigma_{j}(\Theta-M(\lambda)), \quad j \in\{p, c, r\} .
\end{gathered}
$$

2.5. Boundary triplets and Weyl functions for a dual pair $\{A, A\}$. Clearly, every symmetric operator $A$ in $\mathfrak{H}$ generates a dual pair of the form $\{A, A\}$, and vise versa. In this case the definition of a boundary triplet for a general dual pair from [23, 29] (see also Definition 1.1) is simplified and reads as follows.

Definition 2.9. Let $A$ be a closed symmetric operator in $\mathfrak{H}$ with equal deficiency indices $n_{+}(A)=n_{-}(A) \leq \infty$. A collection $\Pi=\left\{\mathcal{H} \oplus \mathcal{H}, \Gamma, \Gamma^{\top}\right\}$, where $\mathcal{H}$ is a Hilbert space and

$$
\Gamma=\left(\begin{array}{l}
\Gamma_{0} \\
\Gamma_{1}
\end{array}\right): A^{*} \rightarrow \mathcal{H} \oplus \mathcal{H}, \quad \Gamma^{\top}=\left(\begin{array}{l}
\Gamma_{0}^{\top} \\
\Gamma_{1}^{\top}
\end{array}\right): A^{*} \rightarrow \mathcal{H} \oplus \mathcal{H}
$$

are linear mappings, is called a boundary triplet for $\{A, A\}$ if the mappings $\Gamma$ and $\Gamma^{\top}$ are surjective and the following abstract Green's identity holds

$$
\left(f^{\prime}, g\right)-\left(f, g^{\prime}\right)=\left(\Gamma_{1} \widehat{f}, \Gamma_{0}^{\top} \widehat{g}\right)-\left(\Gamma_{0} \widehat{f}, \Gamma_{1}^{\top} \widehat{g}\right), \quad \widehat{f}=\left\{f, f^{\prime}\right\}, \widehat{g}=\left\{g, g^{\prime}\right\} \in A^{*} .
$$


In the sequel we consider only dual pairs $\{A, A\}$ and specify for this case some results from $[23,29,18]$ on boundary triplets of general dual pairs $\left\{A, A^{\top}\right\}$. Each boundary triplet $\Pi=\left\{\mathcal{H} \oplus \mathcal{H}, \Gamma, \Gamma^{\top}\right\}$ for $\{A, A\}$ satisfies the relations

$$
\operatorname{dim} \mathcal{H}=n_{ \pm}(A) \quad \text { and } \quad \operatorname{ker} \Gamma_{0} \cap \operatorname{ker} \Gamma_{1}=\operatorname{ker} \Gamma_{0}^{\top} \cap \operatorname{ker} \Gamma_{1}^{\top}=A .
$$

With a boundary triplet $\Pi=\left\{\mathcal{H} \oplus \mathcal{H}, \Gamma, \Gamma^{\top}\right\}$ for $\{A, A\}$ one associates two extensions $A_{j}=\operatorname{ker} \Gamma_{j}\left(\in \operatorname{Ext}_{A}\right), j \in\{0,1\}$, which in this case are not necessarily self-adjoint. In what follows it is always assumed that $\rho\left(A_{0}\right) \neq \emptyset$.

The following result is a counterpart of Proposition 2.5 for dual pairs $\{A, A\}$.

Proposition 2.10. Let $\Pi=\left\{\mathcal{H} \oplus \mathcal{H}, \Gamma, \Gamma^{\top}\right\}$ be a boundary triplet for $\{A, A\}$. Then the mapping (2.8) establishes a bijective correspondence between the sets $\operatorname{Ext}_{A}$ and $\widetilde{\mathcal{C}}(\mathcal{H})$. Moreover, with $\Theta:=B \in \mathcal{C}(\mathcal{H})$ the formula (2.8) takes the form (2.9).

We usually indicate the correspondence in Proposition 2.10 as $\widetilde{A}=A_{\Theta}$. The linear relation $\Theta$ is called the boundary relation or the coordinate, of the proper extension $\widetilde{A}=A_{\Theta}$ in the triplet $\Pi$.

Definition 2.11. Let $\Pi=\left\{\mathcal{H} \oplus \mathcal{H}, \Gamma, \Gamma^{\top}\right\}$ be a boundary triplet for $\{A, A\}$. The operator functions $\gamma_{\Pi}(\cdot): \rho\left(A_{0}\right) \rightarrow[\mathcal{H}, \mathfrak{H}]$ and $M_{\Pi}(\cdot): \rho\left(A_{0}\right) \rightarrow[\mathcal{H}]$ defined by

$$
\gamma_{\Pi}(z):=\pi_{1}\left(\Gamma_{0} \uparrow \widehat{\mathfrak{N}}_{z}(A)\right)^{-1}, \quad \Gamma_{1} \uparrow \widehat{\mathfrak{N}}_{z}(A)=M_{\Pi}(z) \Gamma_{0} \uparrow \widehat{\mathfrak{N}}_{z}(A)
$$

with $z \in \rho\left(A_{0}\right) \neq \emptyset$ are called, respectively, the $\gamma$-field and the Weyl function corresponding to $\Pi$.

In other words the Weyl function $M_{\Pi}(\cdot)$ is defined as follows (cf. (2.10))

$$
\Gamma_{1}\left\{f_{z}, z f_{z}\right\}=M_{\Pi}(z) \Gamma_{0}\left\{f_{z}, z f_{z}\right\}, \quad f_{z} \in \operatorname{ker}\left(A^{*}-z\right), \quad z \in \rho\left(A_{0}\right) .
$$

The functions $\gamma_{\Pi}(\cdot)$ and $M_{\Pi}(\cdot)$ are well defined and holomorphic on $\rho\left(A_{0}\right)$.

Each boundary triplet $\Pi=\left\{\mathcal{H} \oplus \mathcal{H}, \Gamma, \Gamma^{\top}\right\}$ generates a (reversed) boundary triplet $\Pi_{\top}=\left\{\mathcal{H} \oplus \mathcal{H}, \Gamma_{\top},\left(\Gamma_{\top}\right)^{\top}\right\}$ for $\{A, A\}$, which is defined by

$$
\Gamma_{\top}=\Gamma^{\top}=\left(\begin{array}{l}
\Gamma_{0}^{\top} \\
\Gamma_{1}^{\top}
\end{array}\right): A^{*} \rightarrow \mathcal{H} \oplus \mathcal{H} \quad \text { and } \quad\left(\Gamma_{\top}\right)^{\top}=\Gamma=\left(\begin{array}{l}
\Gamma_{0} \\
\Gamma_{1}
\end{array}\right): A^{*} \rightarrow \mathcal{H} \oplus \mathcal{H} .
$$

For this triplet one has $A_{0 \top}\left(=\operatorname{ker} \Gamma_{0}^{\top}\right)=A_{0}^{*}$ and the corresponding $\gamma$-field $\gamma_{\Pi_{\top}}(\cdot)$ : $\rho\left(A_{0}^{*}\right) \rightarrow[\mathcal{H}, \mathfrak{H}]$ and the Weyl function $M_{\Pi_{\top}}(\cdot): \rho\left(A_{0}^{*}\right) \rightarrow[\mathcal{H}]$ are defined for every $z \in \rho\left(A_{0}^{*}\right)$ by

$$
\gamma_{\Pi_{\top}}(z):=\pi_{1}\left(\Gamma_{0}^{\top} \uparrow \widehat{\mathfrak{N}}_{z}(A)\right)^{-1}, \quad \Gamma_{1}^{\top} \uparrow \widehat{\mathfrak{N}}_{z}(A)=M_{\Pi_{\top}}(z) \Gamma_{0}^{\top} \uparrow \widehat{\mathfrak{N}}_{z}(A) .
$$

Remark 2.12. In the case that $\Gamma^{\top}=\Gamma=\left(\begin{array}{c}\Gamma_{0} \\ \Gamma_{1}\end{array}\right)$ the boundary triplet $\Pi=\left\{\mathcal{H} \oplus \mathcal{H}, \Gamma, \Gamma^{\top}\right\}$ for the dual pair $\{A, A\}$ turns into the boundary triplet $\Pi=\left\{\mathcal{H}, \Gamma_{0}, \Gamma_{1}\right\}$ for $A^{*}$ in Definition 2.3, while the Weyl function $M_{\Pi}(\cdot)$ given by (2.12) becomes the Weyl function $M(\cdot)$ defined in (2.10). This remark shows that a boundary triplet $\left\{\mathcal{H}, \Gamma_{0}, \Gamma_{1}\right\}$ for $A^{*}$ in the sense of Definition 2.3 can be regarded as a particular case of a boundary triplet $\left\{\mathcal{H} \oplus \mathcal{H}, \Gamma, \Gamma^{\top}\right\}$ for $\{A, A\}$ in the sense of Definition 2.9. To distinguish between two kinds of boundary triplets, the triplet $\Pi=\left\{\mathcal{H}, \Gamma_{0}, \Gamma_{1}\right\}$ for $A^{*}$ will sometimes be called an ordinary boundary triplet.

2.6. Unitary equivalent boundary triplets. Let $\mathfrak{H}_{1}$ and $\mathfrak{H}_{2}$ be Hilbert spaces. For each unitary operator $U \in\left[\mathfrak{H}_{1}, \mathfrak{H}_{2}\right]$ from $\mathfrak{H}_{1}$ onto $\mathfrak{H}_{2}$ denote $\widetilde{U}:=U \oplus U \in\left[\mathfrak{H}_{1} \oplus \mathfrak{H}_{1}, \mathfrak{H}_{2} \oplus\right.$ $\left.\mathfrak{H}_{2}\right]$. 
Definition 2.13. The linear relations $T_{1} \in \widetilde{\mathcal{C}}\left(\mathfrak{H}_{1}\right)$ and $T_{2} \in \widetilde{\mathcal{C}}\left(\mathfrak{H}_{2}\right)$ are said to be unitarily equivalent by means of the unitary operator $U \in\left[\mathfrak{H}_{1}, \mathfrak{H}_{2}\right]$, if

$$
T_{2}=\widetilde{U} T_{1}\left(=\left\{\left\{U f, U f^{\prime}\right\}:\left\{f, f^{\prime}\right\} \in T_{1}\right\}\right) .
$$

Definition 2.14. Let $A^{(j)}$ be a closed symmetric operator in $\mathfrak{H}_{j}, j \in\{1,2\}$, and let $\Pi_{j}=\left\{\mathcal{H} \oplus \mathcal{H}, \Gamma^{(j)}, \Gamma^{\top(j)}\right\}$ be a boundary triplet for $\left\{A^{(j)}, A^{(j)}\right\}$. The boundary triplets $\Pi_{1}$ and $\Pi_{2}$ are said to be unitarily equivalent (unitarily similar) by means of the unitary operator $U \in\left[\mathfrak{H}_{1}, \mathfrak{H}_{2}\right]$, if

$$
\widetilde{U} A^{(1) *}=A^{(2) *} \quad \text { and } \quad \Gamma^{(2)} \widetilde{U} \uparrow A^{(1) *}=\Gamma^{(1)},
$$

The boundary triplets $\Pi_{1}$ and $\Pi_{2}$ are said to be unitarily similar if they are unitarily similar by means of some unitary operator $U \in\left[\mathfrak{H}_{1}, \mathfrak{H}_{2}\right]$.

The following lemma is immediate from Definition 2.14.

Lemma 2.15. Let the boundary triplets $\Pi_{1}$ and $\Pi_{2}$ for $\left\{A^{(1)}, A^{(1)}\right\}$ and $\left\{A^{(2)}, A^{(2)}\right\}$, respectively, be unitarily similar by means of $U$. Then for any $\Theta \in \widetilde{\mathcal{C}}(\mathcal{H})$ the corresponding proper extensions $A_{\Theta}^{(1)} \in \operatorname{Ext}_{A^{(1)}}$ and $A_{\Theta}^{(2)} \in \operatorname{Ext}_{A^{(2)}}$ are also unitarily equivalent by means of the same $U$. In particular, for any $B \in[\mathcal{H}]$ the extensions $A_{B}^{(j)}=\operatorname{ker}\left(\Gamma_{1}^{(j)}-B \Gamma_{0}^{(j)}\right), j \in\{1,2\}$, are unitarily similar by means of $U$.

Note that in the case $\Gamma^{(j)}=\Gamma^{\top(j)}, j \in\{1,2\}$, Definition 2.14 coincides with the usual definition of unitary equivalence of ordinary boundary triplets $\Pi_{j}=\left\{\mathcal{H}, \Gamma_{0}^{(j)}, \Gamma_{1}^{(j)}\right\}$ for $A^{(j) *}$. Moreover, in this case the following theorem holds.

Theorem 2.16. $[12,14]$ Let $A^{(j)}$ be a simple symmetric operator in $\mathfrak{H}_{j}$, let $\Pi_{j}=$ $\left\{\mathcal{H}, \Gamma_{0}^{(j)}, \Gamma_{1}^{(j)}\right\}$ be a boundary triplet for $A^{(j) *}$ and let $M_{j}(\cdot)$ be the corresponding Weyl function, $j \in\{1,2\}$. Then the boundary triplets $\Pi_{1}$ and $\Pi_{2}$ are unitarily equivalent if and only if

$$
M_{1}(z)=M_{2}(z), \quad z \in \mathbb{C}_{+}
$$

\section{UNITARY EQUIVALENCE OF PROPER EXTENSIONS}

3.1. Basic lemma. Let $A$ be a closed symmetric, not necessarily densely defined, operator in $\mathfrak{H}$ with equal deficiency indices $n_{+}(A)=n_{-}(A) \leq \infty$. If $A$ is simple, then according to Theorem 2.16 and Lemma 2.15, the Weyl function $M(\cdot)$ of an ordinary boundary triplet $\Pi=\left\{\mathcal{H}, \Gamma_{0}, \Gamma_{1}\right\}$ for $A^{*}$ determines the relations $A, A^{*}, A_{0}, A_{1}$ uniquely up to unitary equivalence.

In what follows we consider the following transform

$$
\widetilde{M}(z):=K^{*}(B-M(z))^{-1} K
$$

of the Weyl function $M(\cdot)$ with bounded, not necessarily self-adjoint, operator $B$. We investigate whether the function $\widetilde{M}(\cdot)$ determines the operator $A$ and its proper extensions uniquely up to unitary equivalence. It will be shown that in general this is not the case, although it is really true under some additional assumptions on the relation $A_{0}=\operatorname{ker} \Gamma_{0}$.

We start with the following basic lemma.

Lemma 3.1. Let $F_{j}(\cdot) \in R\left[\mathcal{H}_{j}\right]$ be an $R$-function and let $\Sigma_{j}(\cdot), C_{j}, D_{j} j \in\{1,2\}$, be its spectral measure and the parameters, respectively (see (2.3)), i.e.,

$$
F_{j}(z)=C_{j}+D_{j} z+\int_{\mathbb{R}}\left(\frac{1}{t-z}-\frac{t}{1+t^{2}}\right) d \Sigma_{j}, \quad j \in\{1,2\} .
$$


Moreover, let $\mathcal{H}$ be a Hilbert space, let $B_{j} \in\left[\mathcal{H}_{j}\right]$ and $K_{j} \in\left[\mathcal{H}, \mathcal{H}_{j}\right], j \in\{1,2\}$, be operators with $0 \in \rho\left(K_{1}\right) \cap \rho\left(K_{2}\right)$ and such that the set

$$
\Omega_{+}:=\left\{z \in \mathbb{C}_{+}: 0 \in \rho\left(B_{1}-F_{1}(z)\right) \cap \rho\left(B_{2}-F_{2}(z)\right)\right\}
$$

is not empty, and let the operator measure $\widetilde{\Sigma}_{j}(\cdot): \mathcal{B}_{b}(\mathbb{R}) \rightarrow[\mathcal{H}]$ and the operators $\widetilde{C}_{j}, \widetilde{D}_{j}, \widetilde{B}_{j} \in[\mathcal{H}]$ be given by

$$
\begin{gathered}
\widetilde{\Sigma}_{j}(\delta):=K_{j}^{-1} \Sigma_{j}(\delta) K_{j}^{-1 *}, \quad \delta \in \mathcal{B}_{b}(\mathbb{R}), \\
\widetilde{C}_{j}:=K_{j}^{-1} C_{j} K_{j}^{-1 *}, \quad \widetilde{D}_{j}:=K_{j}^{-1} D_{j} K_{j}^{-1 *}, \quad \widetilde{B}_{j}:=K_{j}^{-1} B_{j} K_{j}^{-1 *} .
\end{gathered}
$$

Then the equality

$$
K_{1}^{*}\left(B_{1}-F_{1}(z)\right)^{-1} K_{1}=K_{2}^{*}\left(B_{2}-F_{2}(z)\right)^{-1} K_{2}, \quad z \in \Omega_{+},
$$

implies the following equalities:

$$
\begin{gathered}
\widetilde{\Sigma}_{1}^{s}(\delta)=\widetilde{\Sigma}_{2}^{s}(\delta), \quad \widetilde{\Sigma}_{2}^{a c}(\delta)-\widetilde{\Sigma}_{1}^{a c}(\delta)=\frac{m(\delta)}{\pi}\left(\operatorname{Im} \widetilde{B}_{2}-\operatorname{Im} \widetilde{B}_{1}\right), \quad \delta \in \mathcal{B}_{b}(\mathbb{R}), \\
\widetilde{C}_{1}-\operatorname{Re} \widetilde{B}_{1}=\widetilde{C}_{2}-\operatorname{Re} \widetilde{B}_{2}, \quad \widetilde{D}_{1}=\widetilde{D}_{2} .
\end{gathered}
$$

Proof. Starting with (3.6) and taking inverses we get

$$
K_{1}^{-1}\left(B_{1}-F_{1}(z)\right)\left(K_{1}^{*}\right)^{-1}=K_{2}^{-1}\left(B_{2}-F_{2}(z)\right)\left(K_{2}^{*}\right)^{-1}, \quad z \in \Omega_{+} .
$$

Since $F_{1}(\cdot)$ and $F_{2}(\cdot)$ are Nevanlinna functions, the equality (3.9) remains valid for all $z \in \mathbb{C}_{+}$by continuity. Substituting the integral representations (3.2) in (3.9) one obtains

$$
\begin{aligned}
& \widetilde{C}_{1}-\widetilde{B}_{1}+\widetilde{D}_{1} z+\int_{\mathbb{R}}\left(\frac{1}{t-z}-\frac{t}{1+t^{2}}\right) d \widetilde{\Sigma}_{1} \\
& =\widetilde{C}_{2}-\widetilde{B}_{2}+\widetilde{D}_{2} z+\int_{\mathbb{R}}\left(\frac{1}{t-z}-\frac{t}{1+t^{2}}\right) d \widetilde{\Sigma}_{2}, \quad z \in \mathbb{C}_{+} .
\end{aligned}
$$

Dividing both sides of (3.10) by $z$ and passing to the limit as $z \rightarrow \infty$ gives

$$
\widetilde{D}_{1}=\widetilde{D}_{2} .
$$

Now by taking $z=i$ in (3.10) leads to

$$
\widetilde{C}_{1}-\widetilde{B}_{1}+i \int_{\mathbb{R}} \frac{d \widetilde{\Sigma}_{1}}{1+t^{2}}=\widetilde{C}_{2}-\widetilde{B}_{2}+i \int_{\mathbb{R}} \frac{d \widetilde{\Sigma}_{2}}{1+t^{2}} .
$$

Since $\widetilde{C}_{i}=\widetilde{C}_{j}^{*}, j \in\{1,2\}$, the equality (3.12) gives $\widetilde{C}_{1}-\operatorname{Re} \widetilde{B}_{1}=\widetilde{C}_{2}-\operatorname{Re} \widetilde{B}_{2}$.

On the other hand, by taking the imaginary parts on both sides of (3.10) and subtracting the terms in (3.11) one obtains for all $y>0$ and $x \in \mathbb{R}$,

$$
-\operatorname{Im} \widetilde{B}_{1}+\int_{\mathbb{R}} \frac{y}{(t-x)^{2}+y^{2}} d \widetilde{\Sigma}_{1}=-\operatorname{Im} \widetilde{B}_{2}+\int_{\mathbb{R}} \frac{y}{(t-x)^{2}+y^{2}} d \widetilde{\Sigma}_{2} .
$$

For a given $h \in \mathcal{H}$ denote by $\mu_{j, h}$ the scalar measure

$$
\mu_{j, h}(\delta)=\left(\widetilde{\Sigma}_{j}(\delta) h, h\right), \quad \delta \in \mathcal{B}_{b}(\mathbb{R}), \quad j \in\{1,2\},
$$

and let $\mu_{j, h}(t)$ be the corresponding scalar distribution function (see (2.5)). Since $\widetilde{\Sigma}_{j}$ satisfies (2.4), it follows that

$$
\int_{\mathbb{R}} \frac{d \mu_{j, h}}{1+t^{2}}<\infty, \quad h \in \mathcal{H}, \quad j \in\{1,2\} .
$$

Moreover, (3.13) implies that for every $h \in \mathcal{H}, y>0$ and $x \in \mathbb{R}$,

$$
-\left(\operatorname{Im} \widetilde{B}_{1} h, h\right)+\int_{\mathbb{R}} \frac{y}{(t-x)^{2}+y^{2}} d \mu_{1, h}=-\left(\operatorname{Im} \widetilde{B}_{2} h, h\right)+\int_{\mathbb{R}} \frac{y}{(t-x)^{2}+y^{2}} d \mu_{2, h} .
$$


Let $X_{j, h}$ be the set of all $x \in \mathbb{R}$ for which the derivative $\frac{d \mu_{j, h}(x)}{d x}$ exists, $j \in\{1,2\}$. Since the measure $\mu_{j, h}$ satisfies (3.15), we can pass to the limit in (3.16) as $y \downarrow 0$. Applying the Fatou theorem, we arrive at the basic equality

$$
-\left(\operatorname{Im} \widetilde{B}_{1} h, h\right)+\pi \frac{d \mu_{1, h}(t)}{d t}=-\left(\operatorname{Im} \widetilde{B}_{2} h, h\right)+\pi \frac{d \mu_{2, h}(t)}{d t},
$$

which holds for every $h \in \mathcal{H}$ and $t \in X_{1, h} \cap X_{2, h}$. Let $\frac{d \mu_{j, h}^{a c}}{d m}(\cdot)$ be the derivative of the (absolutely continuous) measure $\mu_{j, h}^{a c}$ with respect to the Lebesgue measure $m$. Since

$$
\frac{d \mu_{j, h}^{a c}}{d m}(t)=\frac{d \mu_{j, h}(t)}{d t} \text { for a.e. } t \in \mathbb{R}, \quad j \in\{1,2\},
$$

it follows from (3.17) that

$$
\mu_{2, h}^{a c}(\delta)-\mu_{1, h}^{a c}(\delta)=\frac{m(\delta)}{\pi}\left(\left(\operatorname{Im} \widetilde{B}_{2}-\operatorname{Im} \widetilde{B}_{1}\right) h, h\right), \quad \delta \in \mathcal{B}_{b}(\mathbb{R}), \quad h \in \mathcal{H} .
$$

Combining (3.18) with (3.14) one obtains the second equality in (3.7). Substituting this equality in (3.13) and using the Lebesgue decomposition $\Sigma_{j}(\cdot)=\Sigma_{j}^{a c}(\cdot)+\Sigma_{j}^{s}(\cdot)($ see $(2.2))$ leads to

$$
\int_{\mathbb{R}} \frac{y}{(t-x)^{2}+y^{2}} d \widetilde{\Sigma}_{1}^{s}=\int_{\mathbb{R}} \frac{y}{(t-x)^{2}+y^{2}} d \widetilde{\Sigma}_{2}^{s} .
$$

Since the operator-valued measure is uniquely recovered by its Poisson transform (for instance, by means of the Stielties inversion formula), the first equality in (3.7) follows.

Corollary 3.2. Let the conditions of Lemma 3.1 be satisfied and let $\Sigma_{j}(\cdot)$ be the spectral function of $F_{j}(\cdot)($ see $(2.5))$. Assume, in addition, that at least one of the following conditions is fulfilled:

(i) The operator measure $\Sigma_{j}^{a c}(\cdot), j \in\{1,2\}$, is not equivalent to the Lebesgue measure $m(\cdot)$ and equality (3.6) holds.

(ii) The set

$$
\Omega_{-}:=\left\{z \in \mathbb{C}_{-}: 0 \in \rho\left(B_{1}-F_{1}(z)\right) \cap \rho\left(B_{2}-F_{2}(z)\right)\right\}
$$

is not empty and the following equality holds

$$
K_{1}^{*}\left(B_{1}-F_{1}(z)\right)^{-1} K_{1}=K_{2}^{*}\left(B_{2}-F_{2}(z)\right)^{-1} K_{2}, \quad z \in \Omega:=\Omega_{+} \cup \Omega_{-} .
$$

(iii) Equality (3.6) holds and for some $t_{0} \in \mathbb{R}$ the weak derivatives $w-\frac{d \Sigma_{1}}{d t}\left(t_{0}\right), w-\frac{d \Sigma_{2}}{d t}\left(t_{0}\right)$ exist and

$$
w-\frac{d \Sigma_{1}}{d t}\left(t_{0}\right)=w-\frac{d \Sigma_{2}}{d t}\left(t_{0}\right)
$$

Then the following relations are valid

$$
\begin{gathered}
\widetilde{\Sigma}_{1}=\widetilde{\Sigma}_{2}, \quad \operatorname{Im} \widetilde{B}_{1}=\operatorname{Im} \widetilde{B}_{2}, \\
\widetilde{C}_{1}-\operatorname{Re} \widetilde{B}_{1}=\widetilde{C}_{2}-\operatorname{Re} \widetilde{B}_{2}, \quad \widetilde{D}_{1}=\widetilde{D}_{2} .
\end{gathered}
$$

Proof. First observe that according to Lemma 3.1 each of the assumptions (i), (ii), (iii) imply relations (3.7) and (3.8).

(i) Since the measure $\Sigma_{j}^{a c}$ is not equivalent to the Lebesgue measure $m(\cdot)$, there exists a bounded Borel set $\delta_{j}$ of positive Lebesgue measure $m\left(\delta_{j}\right)>0$, such that $\Sigma_{j}^{a c}\left(\delta_{j}\right)=0$; $j \in\{1,2\}$. Substituting these sets in the second equality in (3.7) we get

$$
\operatorname{Im} \widetilde{B}_{2}-\operatorname{Im} \widetilde{B}_{1}=-\frac{\pi}{m\left(\delta_{2}\right)} \widetilde{\Sigma}_{1}^{a c}\left(\delta_{2}\right) \leq 0, \quad \operatorname{Im} \widetilde{B}_{2}-\operatorname{Im} \widetilde{B}_{1}=\frac{\pi}{m\left(\delta_{1}\right)} \widetilde{\Sigma}_{2}^{a c}\left(\delta_{1}\right) \geq 0 .
$$


Hence $\operatorname{Im} \widetilde{B}_{2}=\operatorname{Im} \widetilde{B}_{1}$, and the second equality in (3.7) yields $\widetilde{\Sigma}_{1}^{a c}=\widetilde{\Sigma}_{2}^{a c}$. Combining this equality with the first equality in (3.7) yields $\widetilde{\Sigma}_{1}=\widetilde{\Sigma}_{2}$.

(ii) Starting with equality (3.20) for $z \in \Omega_{-}$and repeating the reasonings of the proof of Lemma 3.1, we arrive at the equality

$$
-\operatorname{Im} \widetilde{B}_{1}+\int_{\mathbb{R}} \frac{y}{(t-x)^{2}+y^{2}} d \widetilde{\Sigma}_{1}=-\operatorname{Im} \widetilde{B}_{2}+\int_{\mathbb{R}} \frac{y}{(t-x)^{2}+y^{2}} d \widetilde{\Sigma}_{2},
$$

with $y<0$ and $x \in \mathbb{R}$. Therefore for any $y>0$ and $x \in \mathbb{R}$ one has

$$
-\operatorname{Im} \widetilde{B}_{1}-\int_{\mathbb{R}} \frac{y}{(t-x)^{2}+y^{2}} d \widetilde{\Sigma}_{1}=-\operatorname{Im} \widetilde{B}_{2}-\int_{\mathbb{R}} \frac{y}{(t-x)^{2}+y^{2}} d \widetilde{\Sigma}_{2} .
$$

Combining this equality with (3.13) and using the uniqueness of the Poisson transform, we arrive at (3.22).

(iii) As it was shown in the proof of Lemma 3.1 equality (3.6) implies the basic equality (3.17). Since $t_{0} \in X_{1, h} \cap X_{2, h}$ for all $h \in \mathcal{H}$, we get from (3.17)

$$
-\left(\operatorname{Im} \widetilde{B}_{1} h, h\right)+\pi \frac{d \mu_{1, h}\left(t_{0}\right)}{d t}=-\left(\operatorname{Im} \widetilde{B}_{2} h, h\right)+\pi \frac{d \mu_{2, h}\left(t_{0}\right)}{d t}, \quad h \in \mathcal{H} .
$$

Combining (3.24) with (3.21) one obtains $\operatorname{Im} \widetilde{B}_{1}=\operatorname{Im} \widetilde{B}_{2}$. Inserting this equality in (3.7), yields the first equality in (3.22).

Remark 3.3. Corollary 3.2(iii) demonstrates at the same time that the condition (i) of this corollary is not necessary for the validity of the statement. In fact, the conclusion of Corollary 3.2 substantially depends on the measures $\Sigma_{j}$ themselves, not only on their spectral types. We emphasize that the assumptions (iii) of Corollary 3.2, hence the conclusions, can be satisfied even if the operator measures $\Sigma_{j}^{a c}, j \in\{1,2\}$, are spectrally equivalent to the operator Lebesgue measure $m_{\mathcal{H}}:=I_{\mathcal{H}} m$ in the sense of [26, Definition 4.8].

Indeed, let $t_{0}$ be a common point for which the weak derivatives $\frac{d \Sigma_{j}}{d t}\left(t_{0}\right), j \in\{1,2\}$, exist. Choose a scalar function $\varphi(\cdot) \in C^{1}(\mathbb{R})$ satisfying

$$
\varphi\left(t_{0}\right)=0, \quad \varphi(t)>0, \quad t \in \mathbb{R} \backslash\left\{t_{0}\right\} \quad \text { and } \quad \varphi(t)=1, \quad t \in \mathbb{R} \backslash\left(t_{0}-1, t_{0}+1\right)
$$

and define the measure $\Sigma_{j, \varphi}$ by setting

$$
\Sigma_{j, \varphi}(\delta):=\int_{\delta} \varphi(t) d \Sigma_{j}(t), \quad \delta \in \mathcal{B}_{b}(\mathbb{R}), \quad j \in\{1,2\}
$$

Then the weak derivative $w-\frac{d \Sigma_{j}, \varphi}{d t}\left(t_{0}\right), j \in\{1,2\}$, exists and equals zero since for every $h \in \mathcal{H}$

$$
\left.\frac{\left(d \Sigma_{j, \varphi}(t) h, h\right)}{d t}\right|_{t=t_{0}}=\left.\varphi\left(t_{0}\right) \frac{\left(d \Sigma_{j}(t) h, h\right)}{d t}\right|_{t=t_{0}}=0, \quad j \in\{1,2\} .
$$

If $\Sigma_{j}^{a c}, j \in\{1,2\}$, are spectrally equivalent to the measure $m_{\mathcal{H}}$, then clearly $\Sigma_{j, \varphi}^{a c} \sim m_{\mathcal{H}}$. Moreover, their multiplicity functions coincide,

$$
N_{\Sigma_{j, \varphi}^{a c}}(t)=N_{m_{\mathcal{H}}}(t)=\operatorname{dim} \mathcal{H}, \quad t \in \mathbb{R} \backslash\left\{t_{0}\right\} .
$$

Hence the operator measures $\Sigma_{j, \varphi}^{a c}$ and $m_{\mathcal{H}}$ are also spectrally equivalent.

Now let $F_{j}, j \in\{1,2\}$, be $R[\mathcal{H}]$-functions as in (3.2) and let $\Sigma_{j}$ be the spectral measure of $F_{j}, j \in\{1,2\}$. Let $K_{1}=K_{2}$ and let $B_{j} \in[\mathcal{H}], j \in\{1,2\}$, be such that the equation (3.6) is satisfied, while $F_{1} \neq F_{2}$; for instance, one can take $F_{2}(z)=F_{1}(z) \pm i I$ with $z \in \mathbb{C}_{ \pm}$and put $B_{1}=0, B_{2}=i I$. Here the operator measures $\Sigma_{j}^{a c}, j \in\{1,2\}$ can be taken to be spectrally equivalent to the measure $m_{\mathcal{H}}$. In this case, the assumptions and the conclusions of Corollary 3.2 fail to hold, while the assumptions in Lemma 3.1 are fulfilled. 
We now slightly modify the previous situation by replacing the operator measures $\Sigma_{j}$ by $\Sigma_{j, \varphi}$ defined in (3.25) and define the $R[\mathcal{H}]$-functions $F_{j, \varphi}$ according to (3.2) with $\Sigma_{j, \varphi}$ instead of $\Sigma_{j}, j \in\{1,2\}$. If now for some $\widetilde{B}_{j} \in[\mathcal{H}], j \in\{1,2\}$, the equality (3.6) with $F_{j, \varphi}$ (instead of $F_{j}, B_{j}$ ) holds, then due to (3.26), and in contrary to the previous case, we have $F_{1, \varphi} \equiv F_{2, \varphi}$ and $\Sigma_{1, \varphi}(t)=\Sigma_{2, \varphi}(t), t \in \mathbb{R}$.

3.2. Sufficient conditions for unitary similarity of ordinary boundary triplets. The results in the previous subsection are now applied to establish the unitary equivalence of proper extensions of $A$ as well as the unitary equivalence of appropriate boundary triplets for $A^{*}$.

Combining Lemma 3.1 with Proposition 2.8 yields the following statement.

Proposition 3.4. Let $A^{(j)}$ be a simple symmetric operator in the separable Hilbert space $\mathfrak{H}_{j}$, let $\Pi_{j}=\left\{\mathcal{H}_{j}, \Gamma_{0}^{(j)}, \Gamma_{1}^{(j)}\right\}$ be a boundary triplet for $A^{(j) *}$ and let $M_{j}(\cdot)$ be the corresponding Weyl function with the integral representation

$$
M_{j}(z)=C_{j}+D_{j} z+\int_{\mathbb{R}}\left(\frac{1}{t-z}-\frac{t}{1+t^{2}}\right) d \Sigma_{j}, \quad j \in\{1,2\},
$$

(see (2.3)). Moreover, let $\mathcal{H}$ be a Hilbert space and let $B_{j} \in\left[\mathcal{H}_{j}\right]$ and $K_{j} \in\left[\mathcal{H}, \mathcal{H}_{j}\right], j \in$ $\{1,2\}$, be operators such that $0 \in \rho\left(K_{1}\right) \cap \rho\left(K_{2}\right)$ and

$$
\Omega_{+}:=\rho\left(A_{B_{1}}^{(1)}\right) \cap \rho\left(A_{B_{2}}^{(2)}\right) \cap \mathbb{C}_{+} \neq \emptyset,
$$

where $A_{B_{j}}^{(j)}\left(\in \operatorname{Ext}_{A^{(j)}}\right)$ is given by (2.9) with the boundary operator $B_{j}, j \in\{1,2\}$. Then the equality

$$
K_{1}^{*}\left(B_{1}-M_{1}(z)\right)^{-1} K_{1}=K_{2}^{*}\left(B_{2}-M_{2}(z)\right)^{-1} K_{2}, \quad z \in \Omega_{+},
$$

yields the identities (3.7) and (3.8), where the operator measure $\widetilde{\Sigma}_{j}(\cdot): \mathcal{B}_{b}(\mathbb{R}) \rightarrow[\mathcal{H}]$ and the operators $\widetilde{C}_{j}, \widetilde{D}_{j} \widetilde{B}_{j} \in[\mathcal{H}]$ are defined by (3.4) and (3.5).

Combining Corollary 3.2 with Proposition 2.7 gives the following result.

Proposition 3.5. Let the conditions of Proposition 3.4 be satisfied, let $A_{0}^{(j)}=\operatorname{ker} \Gamma_{0}^{(j)}$, and let $E_{j}(\cdot)$ be the spectral measure of $A_{0}^{(j)}, j \in\{1,2\}$. Assume, in addition, that at least one of the following assumptions is fulfilled:

(a1) Equality (3.29) holds, mul $A_{0}^{(j)} \neq \mathfrak{H}_{j}, \quad j \in\{1,2\}$, and the spectral measure $E_{j}^{a c}(\cdot), j \in\{1,2\}$, is not equivalent to the Lebesgue measure $m_{\mathcal{H}}(\cdot)$.

(a2) The set

$$
\Omega_{-}:=\rho\left(A_{B_{1}}^{(1)}\right) \cap \rho\left(A_{B_{2}}^{(2)}\right) \cap \mathbb{C}_{-}
$$

is not empty and the following equality holds

$$
K_{1}^{*}\left(B_{1}-M_{1}(z)\right)^{-1} K_{1}=K_{2}^{*}\left(B_{2}-M_{2}(z)\right)^{-1} K_{2}, \quad z \in \Omega:=\Omega_{+} \cup \Omega_{-} .
$$

Then the relations (3.22) and (3.23) are satisfied.

Corollary 3.6. Let the conditions of Proposition 3.4 be satisfied, let $A_{0}^{(j)}=\operatorname{ker} \Gamma_{0}^{(j)}$, $j \in\{1,2\}$, and let the following assumption be fulfilled:

(a3) Equality (3.29) holds, mul $A_{0}^{(j)} \neq \mathfrak{H}_{j}$, and $\sigma_{a c}\left(A_{0}^{(j)}\right) \neq \mathbb{R}, j \in\{1,2\}$.

Then the relations (3.22) and (3.23) are valid.

The following statement is immediate from Corollary 3.2, (iii).

Proposition 3.7. Let the conditions of Proposition 3.4 be satisfied. Assume in addition that the following assumption is fulfilled:

(a4) Equality (3.29) holds and for some $t_{0} \in \mathbb{R}$ the weak derivatives $w-\frac{d \Sigma_{1}}{d t}\left(t_{0}\right), w-\frac{d \Sigma_{2}}{d t}\left(t_{0}\right)$ exist and are equal. Then the relations (3.22) and (3.23) are valid. 
From Propositions 3.5 and 3.7 one can derive some general sufficient conditions on the unitary equivalence of certain proper extensions. However, we can deduce a more general result involving auxiliary boundary triplets. For this purpose the following simple lemma, which is immediate from [14], is needed.

Lemma 3.8. Let $\Pi=\left\{\mathcal{K}, \Gamma_{0}, \Gamma_{1}\right\}$ be a boundary triplet for $A^{*}$ and let $M(\cdot)$ be the corresponding Weyl function. Moreover, let $\mathcal{H}$ be a Hilbert space and let $B \in[\mathcal{K}]$ and $K \in[\mathcal{H}, \mathcal{K}]$ with $0 \in \rho(K)$. Then the transform

$$
\widehat{\Gamma}_{0}=K^{*} \Gamma_{0}, \quad \widehat{\Gamma}_{1}=K^{-1}\left(\Gamma_{1}-(\operatorname{Re} B) \Gamma_{0}\right),
$$

defines an ordinary boundary triplet $\widehat{\Pi}_{K, B}:=\left\{\mathcal{H}, \widehat{\Gamma}_{0}, \widehat{\Gamma}_{1}\right\}$ for $A^{*}$ such that $\widehat{A}_{0}\left(=\operatorname{ker} \widehat{\Gamma}_{0}\right)=$ $A_{0}$, and the corresponding Weyl function is given by

$$
\widehat{M}(\cdot)=K^{-1}(M(\cdot)-\operatorname{Re} B) K^{-1 *} .
$$

Moreover, if $T \in[\mathcal{K}]$ is a boundary operator (coordinate) of $\widetilde{A} \in \operatorname{Ext}_{A}$ in the triplet $\Pi$ (i.e. $\widetilde{A}=A_{T}$, see (2.9)), then the boundary operator of $\widetilde{A}$ in the triplet $\widehat{\Pi}_{K, B}$ is

$$
\widehat{T}=K^{-1}(T-\operatorname{Re} B) K^{-1 *} .
$$

Now we are ready to state the main result regarding the unitary equivalence of the auxiliary boundary triplets defined by (3.30).

Theorem 3.9. Let $A^{(j)}$ be a simple symmetric operator in the separable Hilbert space $\mathfrak{H}_{j}$ and let $\Pi_{j}=\left\{\mathcal{H}_{j}, \Gamma_{0}^{(j)}, \Gamma_{1}^{(j)}\right\}$ be a boundary triplet for $A^{(j) *}$ with the Weyl function $M_{j}(\cdot), j \in\{1,2\}$. Moreover, let $A_{0}^{(j)}=\operatorname{ker} \Gamma_{0}^{(j)}$, let $\mathcal{H}$ be a separable Hilbert space, and let $B_{j} \in\left[\mathcal{H}_{j}\right]$ and $K_{j} \in\left[\mathcal{H}, \mathcal{H}_{j}\right], j \in\{1,2\}$, be operators such that $0 \in \rho\left(K_{1}\right) \cap \rho\left(K_{2}\right)$ and

$$
\Omega_{+}:=\rho\left(A_{B_{1}}^{(1)}\right) \cap \rho\left(A_{B_{2}}^{(2)}\right) \cap \mathbb{C}_{+} \neq \emptyset .
$$

Assume, in addition, that at least one of the assumptions (a1)-(a4) listed in Propositions 3.5, 3.7 and Corollary 3.6 is satisfied. Then the boundary triplets $\widehat{\Pi}_{K_{1}, B_{1}}$ and $\widehat{\Pi}_{K_{2}, B_{2}}$ defined in Lemma 3.8 are unitarily equivalent by means of a unitary operator $U \in\left[\mathfrak{H}_{1}, \mathfrak{H}_{2}\right]$. In particular, the pairs of extensions $\left\{A_{0}^{(1)}, A_{B_{1}}^{(1)}\right\}$ and $\left\{A_{0}^{(2)}, A_{B_{2}}^{(2)}\right\}$ are unitarily equivalent by means of the same $U$.

Proof. According to Propositions 3.5, 3.7 and Corollary 3.6 $\operatorname{Im} \widetilde{B}_{1}=\operatorname{Im} \widetilde{B}_{2}$ and by the last equality in (3.5) one has

$$
K_{1}^{-1}\left(\operatorname{Im} B_{1}\right) K_{1}^{-1 *}=K_{2}^{-1}\left(\operatorname{Im} B_{2}\right) K_{2}^{-1 *} .
$$

Combining this identity with (3.29) one obtains

$$
K_{1}^{-1}\left(M_{1}(z)-\operatorname{Re} B_{1}\right) K_{1}^{-1 *}=K_{2}^{-1}\left(M_{2}(z)-\operatorname{Re} B_{2}\right) K_{2}^{-1 *}, \quad z \in \Omega_{+} .
$$

On the other hand, according to $(3.31)$ the Weyl function $\widehat{M}_{j}(\cdot)$ corresponding to the boundary triplet $\widehat{\Pi}_{K_{j}, B_{j}}$ is

$$
\widehat{M}_{j}(z)=K_{j}^{-1}\left(M_{j}(z)-\operatorname{Re} B_{j}\right) K_{j}^{-1 *}, \quad j \in\{1,2\}, \quad z \in \mathbb{C}_{+} \cup \mathbb{C}_{-} .
$$

Combining this equality with (3.34) yields $\widehat{M}_{1}(z)=\widehat{M}_{2}(z), z \in \Omega_{+}$. By Theorem 2.16, the boundary triplets $\widehat{\Pi}_{K_{1}, B_{1}}$ and $\widehat{\Pi}_{K_{2}, B_{2}}$ are unitarily equivalent by means of a unitary operator $U \in\left[\mathfrak{H}_{1}, \mathfrak{H}_{2}\right]$.

Next, according to (3.32) a boundary operator (coordinate) $\widehat{B}_{j}$ of the extension $\widetilde{A}_{j}:=$ $A_{B_{j}}$ in the boundary triplet $\widehat{\Pi}_{K_{j}, B_{j}}$ is

$$
\widehat{B}_{j}=K_{j}^{-1}\left(B_{j}-\operatorname{Re} B_{j}\right) K_{j}^{-1 *}=i K_{j}^{-1}\left(\operatorname{Im} B_{j}\right) K_{j}^{-1 *}, \quad j \in\{1,2\} .
$$


Hence, by (3.33), $\widehat{B}_{1}=\widehat{B}_{2}$ and according to Lemma 2.15 the extensions $A_{B_{1}}$ and $A_{B_{2}}$ are unitarily equivalent by means of $U$. Moreover, by Lemma 2.15, the extensions $A_{0}^{(1)}$ and $A_{0}^{(2)}$ are also unitarily equivalent by means of $U$, since their boundary relations (coordinates) in the triplets $\widehat{\Pi}_{K_{1}, B_{1}}$ and $\widehat{\Pi}_{K_{2}, B_{2}}$ coincide: $A_{0}^{(1)}=\operatorname{ker} \Gamma_{0}^{(1)}=\operatorname{ker} \widehat{\Gamma}_{0}^{(1)}$ and $A_{0}^{(2)}=\operatorname{ker} \Gamma_{0}^{(2)}=\operatorname{ker} \widehat{\Gamma}_{0}^{(2)}$.

Next it is shown that the function $\widetilde{M}(\cdot)$ defined in $(3.1)$ is the Weyl function of a boundary triplet $\widetilde{\Pi}$ for the dual pair $\{A, A\}$. This statement explains appearance of the function $\widetilde{M}(\cdot)$ in this section and, in fact, has motivated our investigations. The following statement, which is immediate from [29], establishes a key connection between Theorem 3.9 and the theory of boundary triplets for dual pairs.

Proposition 3.10. Let $A$ be a symmetric operator in $\mathfrak{H}$ and let $\Pi=\left\{\mathcal{K}, \Gamma_{0}, \Gamma_{1}\right\}$ be a boundary triplet for $A^{*}$ with the Weyl function $M(\cdot)$. Let $B \in[\mathcal{K}], K \in[\mathcal{H}, \mathcal{K}], 0 \in$ $\rho(K)$, and define the linear mappings

$$
\widetilde{\Gamma}=\left(\begin{array}{c}
\widetilde{\Gamma}_{0} \\
\widetilde{\Gamma}_{1}
\end{array}\right): A^{*} \rightarrow \mathcal{H} \oplus \mathcal{H}, \quad \widetilde{\Gamma}^{\top}=\left(\begin{array}{l}
\widetilde{\Gamma}_{0}^{\top} \\
\widetilde{\Gamma}_{1}^{\top}
\end{array}\right): A^{*} \rightarrow \mathcal{H} \oplus \mathcal{H}
$$

by

$$
\widetilde{\Gamma}_{0}=K^{-1}\left(B \Gamma_{0}-\Gamma_{1}\right), \quad \widetilde{\Gamma}_{1}=K^{*} \Gamma_{0} ; \quad \widetilde{\Gamma}_{0}^{\top}=K^{-1}\left(B^{*} \Gamma_{0}-\Gamma_{1}\right), \quad \widetilde{\Gamma}_{1}^{\top}=K^{*} \Gamma_{0} .
$$

Then $\widetilde{\Pi}=\left\{\mathcal{H} \oplus \mathcal{H}, \widetilde{\Gamma}, \widetilde{\Gamma}^{\top}\right\}$ is a boundary triplet for the dual pair $\{A, A\}$, such that

$$
\widetilde{A}_{0}:=\operatorname{ker} \widetilde{\Gamma}_{0}=\operatorname{ker}\left(\Gamma_{1}-B \Gamma_{0}\right)
$$

and the corresponding Weyl function is

$$
\widetilde{M}(z):=M_{\widetilde{\Pi}}(z)=K^{*}(B-M(z))^{-1} K, \quad z \in \rho\left(\widetilde{A}_{0}\right) .
$$

If, in addition, $B$ is accumulative, $\operatorname{Im} B \leq 0$, then $\mathbb{C}_{+} \subset \rho\left(\widetilde{A}_{0}\right)$ and $\operatorname{Im} \widetilde{M}(z) \geq 0, z \in$ $\mathbb{C}_{+}$. Hence $\widetilde{M}(\cdot)$ admits integral representation $(2.3)$ in $\mathbb{C}_{+}$.

Remark 3.11. Proposition 3.10 allows one to reformulate the statement of Theorem 3.9 on the unitary equivalence of the extensions $A_{B_{1}}^{(1)}$ and $A_{B_{2}}^{(2)}$ in terms of the equality of the Weyl functions corresponding to special boundary triplets for the dual pair $\{A, A\}$. The corresponding routine reformulations are left for the reader.

Remark 3.12. Let $\Pi=\left\{\mathcal{H}, \Gamma_{0}, \Gamma_{1}\right\}$ be an ordinary boundary triplet for $A^{*}$ with the Weyl function $M(\cdot)$. It follows from the results in [29] that the Weyl functions of any boundary triplet $\widetilde{\Pi}=\left\{\mathcal{H} \oplus \mathcal{H}, \widetilde{\Gamma}, \widetilde{\Gamma}^{\top}\right\}$ for the dual pair $\{A, A\}$ is obtained via the linear fractional transform

$$
\widetilde{M}(z)=\left(X_{3}+X_{4} M(z)\right)\left(X_{1}+X_{2} M(z)\right)^{-1}, \quad z \in \rho\left(\widetilde{A}_{0}\right)
$$

where

$$
X=\left(\begin{array}{ll}
X_{1} & X_{2} \\
X_{3} & X_{4}
\end{array}\right): \mathcal{H} \oplus \mathcal{H} \rightarrow \mathcal{H} \oplus \mathcal{H}
$$

is a bounded operator with bounded inverse.

If, in addition, $0 \in \rho\left(X_{2}\right)$ and $X_{3}=X_{4} X_{2}^{-1} X_{1}-X_{2}^{-1 *}$, then the equality (3.37) takes the form

$$
\widetilde{M}(z)=C+K^{*}(B-M(z))^{-1} K, \quad z \in \rho\left(\widetilde{A}_{0}\right) .
$$

with $K=-X_{2}^{-1}, B=-X_{2}^{-1} X_{1}$, and $C=X_{4} X_{2}^{-1}$. 
We have no general analog of Theorem 3.9 for Weyl functions of the more general form (3.39) or (3.37). Moreover, we have no inner characterization of the Weyl functions of the form (3.36) or (3.39).

\section{UNITARY EQUIVALENT BOUNDARY TRIPLETS FOR SYMMETRIC DUAL PAIRS}

In this section we investigate unitary equivalence of some boundary triplets for the dual pair $\{A, A\}$ in terms of the corresponding Weyl functions.

Assume that $A \in[\mathcal{D}, \mathfrak{H}]$ is a bounded symmetric operator with the closed domain $\mathcal{D} \subset \mathfrak{H}$ and let

$$
\mathfrak{N}=\mathfrak{H} \ominus \mathcal{D}\left(=\operatorname{mul} A^{*}\right), \quad \widehat{\mathfrak{N}}=\{\{0, n\}: n \in \mathfrak{N}\}\left(\subset A^{*}\right)
$$

Consider the block-matrix representation of $A$,

$$
A=\left(\begin{array}{l}
A_{00} \\
A_{10}
\end{array}\right): \mathcal{D} \rightarrow \mathfrak{H}=\mathcal{D} \oplus \mathfrak{N}
$$

A proper extension $\widetilde{A} \in \operatorname{Ext}_{A}$ is called bounded if $\widetilde{A} \in[\mathfrak{H}]$. Every bounded extension $\widetilde{A} \in \operatorname{Ext}_{A}$ admits a block-matrix representation

$$
\widetilde{A}=\left(\begin{array}{cc}
A_{00} & A_{10}^{*} \\
A_{10} & B
\end{array}\right): \mathcal{D} \oplus \mathfrak{N} \rightarrow \mathcal{D} \oplus \mathfrak{N}
$$

with some $B \in[\mathfrak{N}]$ and vise versa. The adjoint $A^{*}$ of $A$ is multivalued and with any bounded extension $\widetilde{A}$ it admits the graph decomposition

$$
A^{*}=\widetilde{A} \dot{+} \widehat{\mathfrak{N}},
$$

where $\dot{+}$ stands for the direct sum of the graphs; cf. [18, Lemma 5.2].

Proposition 4.1. [18] Let $A \in[\mathcal{D}, \mathfrak{H}]$ be a bounded symmetric operator, let $\Pi=\{\mathcal{H} \oplus$ $\left.\mathcal{H}, \Gamma, \Gamma^{\top}\right\}$ be boundary triplet for $\{A, A\}$ such that $A_{0}\left(=\operatorname{ker} \Gamma_{0}\right)$ is a bounded extension of $A$ and let $\pi_{2}$ be the orthoprojector in $\mathfrak{H} \oplus \mathfrak{H}$ onto $\{0\} \oplus \mathfrak{H}$. Then:

(i) The operators $\Gamma_{0} \uparrow \widehat{\mathfrak{N}}$ and $\Gamma_{0}^{\top} \uparrow \widehat{\mathfrak{N}}$ isomorphically map $\widehat{\mathfrak{N}}$ onto $\mathcal{H}$, so that the operators

$$
\begin{gathered}
\gamma_{\Pi}=\pi_{2}\left(\Gamma_{0}\lceil\widehat{\mathfrak{N}})^{-1}, \quad \gamma_{\Pi_{\top}}=\pi_{2}\left(\Gamma_{0}^{\top} \uparrow \widehat{\mathfrak{N}}\right)^{-1}\right. \\
\Gamma_{1} \uparrow \widehat{\mathfrak{N}}=\mathcal{F}_{\Pi} \Gamma_{0} \uparrow \widehat{\mathfrak{N}}
\end{gathered}
$$

are well defined and $\gamma_{\Pi} \in[\mathcal{H}, \mathfrak{N}], \gamma_{\Pi_{\top}} \in[\mathcal{H}, \mathfrak{N}]$, and $\mathcal{F}_{\Pi} \in[\mathcal{H}]$.

(ii) The corresponding Weyl function $M_{\Pi}(\cdot)$ is given by

$$
M_{\Pi}(z)=\mathcal{F}_{\Pi}+\gamma_{\Pi_{\top}}^{*}\left(A_{0}-z\right)^{-1} \gamma_{\Pi}, \quad z \in \rho\left(A_{0}\right) .
$$

Recall $([14,24,29,18])$ that the operator $\mathcal{F}_{\Pi}$ in $(4.5)$ is called a forbidden operator corresponding to $\Pi$.

Next assume that $A \in[\mathcal{D}, \mathfrak{H}]$ is a bounded symmetric operator with the closed domain $\mathcal{D} \subset \mathfrak{H}$ and let $\mathfrak{N}$ and $\widehat{\mathfrak{N}}$ be the subspaces defined in (4.1).

Definition 4.2. A boundary triplet $\Pi=\left\{\mathcal{H} \oplus \mathcal{H}, \Gamma, \Gamma^{\top}\right\}$ for $\{A, A\}$ is said to belong to the class $B T_{\infty}$ if $A_{0}\left(=\operatorname{ker} \Gamma_{0}\right) \in[\mathfrak{H}]$ and $\Gamma_{0}\{0, n\}=\Gamma_{0}^{\top}\{0, n\}, n \in \mathfrak{N}$; in view of (4.4) this latter condition is equivalent to the equality

$$
\gamma_{\Pi}=\gamma_{\Pi_{\top}}=: \gamma
$$

Next it is shown that for each boundary triplet $\Pi=\left\{\mathcal{H} \oplus \mathcal{H}, \Gamma, \Gamma^{\top}\right\}$ from the class $B T_{\infty}$ the linear mappings $\Gamma$ and $\Gamma^{\top}$ can explicitly be expressed by means of the operators $A_{0}, \gamma_{\Pi}, \gamma_{\Pi_{\top}}$, and $\mathcal{F}_{\Pi}$ defined in Proposition 4.1 . 
Lemma 4.3. Let $A \in[\mathcal{D}, \mathfrak{H}]$ be a bounded symmetric operator in $\mathfrak{H}$ and let $\Pi=\{\mathcal{H} \oplus$ $\left.\mathcal{H}, \Gamma, \Gamma^{\top}\right\} \in B T_{\infty}$ be a boundary triplet for $\{A, A\}$. Moreover, let $A_{0}=\operatorname{ker} \Gamma_{0}$, let $\mathcal{F}_{\Pi} \in[\mathcal{H}]$ be the forbidden operator (4.5) and let $\gamma \in[\mathcal{H}, \mathfrak{N}]$ be the operator defined by (4.7). Then the operators $\Gamma_{j}$ and $\Gamma_{j}^{\top}$ admit the representations

$$
\begin{aligned}
\Gamma_{0}\left\{f, f^{\prime}\right\} & =\gamma^{-1}\left(f^{\prime}-A_{0} f\right), & & \Gamma_{1}\left\{f, f^{\prime}\right\}=-\gamma^{*} P_{\mathfrak{N}} f+\mathcal{F}_{\Pi} \gamma^{-1}\left(f^{\prime}-A_{0} f\right), \\
\Gamma_{0}^{\top}\left\{f, f^{\prime}\right\} & =\gamma^{-1}\left(f^{\prime}-A_{0}^{*} f\right), & & \Gamma_{1}^{\top}\left\{f, f^{\prime}\right\}=-\gamma^{*} P_{\mathfrak{N}} f+\mathcal{F}_{\Pi}^{*} \gamma^{-1}\left(f^{\prime}-A_{0}^{*} f\right),
\end{aligned}
$$

where $\left\{f, f^{\prime}\right\} \in A^{*}$.

Conversely, let $\mathfrak{H}$ and $\mathcal{H}$ be Hilbert spaces, let $A \in[\mathcal{D}, \mathfrak{H}](\mathcal{D} \subset \mathfrak{H})$ be a bounded symmetric operator, let $\widetilde{A} \in[\mathfrak{H}]$ be a bounded extension of $A$, let $K$ be an isomorphism from $\mathcal{H}$ onto $\mathfrak{N}(=\mathfrak{H} \ominus \mathcal{D})$, and let $F \in[\mathcal{H}]$. Then the operators $\Gamma=\left(\begin{array}{l}\Gamma_{0} \\ \Gamma_{1}\end{array}\right)$ and $\Gamma^{\top}=\left(\begin{array}{l}\Gamma_{0}^{\top} \\ \Gamma_{1}^{\top}\end{array}\right)$ defined for all $\left\{f, f^{\prime}\right\} \in A^{*}$ by

$$
\begin{array}{cc}
\Gamma_{0}\left\{f, f^{\prime}\right\}=K^{-1}\left(f^{\prime}-\widetilde{A} f\right), & \Gamma_{1}\left\{f, f^{\prime}\right\}=-K^{*} P_{\mathfrak{N}} f+F K^{-1}\left(f^{\prime}-\widetilde{A} f\right), \\
\Gamma_{0}^{\top}\left\{f, f^{\prime}\right\}=K^{-1}\left(f^{\prime}-\widetilde{A}^{*} f\right), & \Gamma_{1}^{\top}\left\{f, f^{\prime}\right\}=-K^{*} P_{\mathfrak{N}} f+F^{*} K^{-1}\left(f^{\prime}-\widetilde{A}^{*} f\right)
\end{array}
$$

form the boundary triplet $\Pi=\left\{\mathcal{H} \oplus \mathcal{H}, \Gamma, \Gamma^{\top}\right\} \in B T_{\infty}$ for $\{A, A\}$. Moreover,

$$
A_{0}=\widetilde{A}, \quad \gamma=K, \quad \mathcal{F}_{\Pi}=F .
$$

Proof. Applying the identity (2.11) to the elements $\left\{f, A_{0} f\right\},\{0, \gamma h\} \in A^{*}$, see (4.3), (4.4), one obtains

$$
-(f, \gamma h)=\left(\Gamma_{1}\left\{f, A_{0} f\right\}, h\right), \quad f \in \mathfrak{H}, \quad h \in \mathcal{H}
$$

Hence,

$$
\Gamma_{1}\left\{f, A_{0} f\right\}=-\gamma^{*} P_{\mathfrak{N}} f, \quad f \in \mathfrak{H} .
$$

Moreover, (4.3) shows that every $\left\{f, f^{\prime}\right\} \in A^{*}$ can be uniquely decomposed as

$$
\left\{f, f^{\prime}\right\}=\left\{f, A_{0} f\right\}+\{0, n\}
$$

with $n=f^{\prime}-A_{0} f \in \mathfrak{N}$. Hence, by applying $\Gamma_{0}$ and $\Gamma_{1}$ to the equality (4.14) and taking (4.13), (4.4) and (4.5) into account one obtains

$$
\begin{gathered}
\Gamma_{0}\left\{f, f^{\prime}\right\}=\Gamma_{0}\left\{f, A_{0} f\right\}+\Gamma_{0}\{0, n\}=0+\gamma_{\Pi}^{-1} n=\gamma^{-1} n=\gamma^{-1}\left(f^{\prime}-A_{0} f\right), \\
\Gamma_{1}\left\{f, f^{\prime}\right\}=\Gamma_{1}\left\{f, A_{0} f\right\}+\Gamma_{1}\{0, n\}=-\gamma^{*} P_{\mathfrak{N}} f+\mathcal{F}_{\Pi} \gamma_{\Pi}^{-1} n \\
=-\gamma^{*} P_{\mathfrak{N}} f+\mathcal{F}_{\Pi} \gamma^{-1}\left(f^{\prime}-A_{0} f\right), \quad\left\{f, f^{\prime}\right\} \in A^{*} .
\end{gathered}
$$

Thus (4.8) is valid. Moreover, the equalities (4.9) hold, since they are analogs of (4.8) for the triplet $\Pi_{\top}$.

Conversely, let the operators $\Gamma_{j}$ and $\Gamma_{j}^{\top}, j \in\{0,1\}$, be defined by (4.10) and (4.11). Then it is immediately checked that $\Gamma A^{*}=\Gamma^{\top} A^{*}=\mathcal{H} \oplus \mathcal{H}$ and the identity (2.11) is satisfied. Hence $\Pi=\left\{\mathcal{H} \oplus \mathcal{H}, \Gamma, \Gamma^{\top}\right\}$ with operators (4.10) and (4.11) is a boundary triplet for $\{A, A\}$. Moreover, the equalities (4.12) are implied by (4.10) and (4.11).

Next a class of boundary triplets analogous to that appearing in Definition 4.2 is introduced with a finite real point (instead of $\infty$ ). Here $A$ is a, not necessarily bounded or densely defined, symmetric operator in $\mathfrak{H}$ such that $\lambda_{0} \in \widehat{\rho}(A) \cap \mathbb{R} \neq \emptyset$.

Definition 4.4. A boundary triplet $\Pi=\left\{\mathcal{H} \oplus \mathcal{H}, \Gamma, \Gamma^{\top}\right\}$ for $\{A, A\}$ is said to belong to the class $B T_{\lambda_{0}}, \lambda_{0} \in \mathbb{R}$, if $\lambda_{0} \in \rho\left(A_{0}\right)$ and

$$
\Gamma_{0}\left\{f, \lambda_{0} f\right\}=\Gamma_{0}^{\top}\left\{f, \lambda_{0} f\right\}, \quad f \in \mathfrak{N}_{\lambda_{0}}\left(=\operatorname{ker}\left(A^{*}-\lambda_{0}\right)\right) .
$$


The next lemma provides a connection between the classes $B T_{\lambda_{0}}$ and $B T_{\infty}$.

Lemma 4.5. Let $A$ be a symmetric operator in $\mathfrak{H}$ with $\lambda_{0}=\bar{\lambda}_{0} \in \widehat{\rho}(A)$, let $\Pi=$ $\left\{\mathcal{H} \oplus \mathcal{H}, \Gamma, \Gamma^{\top}\right\} \in B T_{\lambda_{0}}$ be a boundary triplet for $\{A, A\}$, let $A_{0}=\operatorname{ker} \Gamma_{0}$ and let $M_{\Pi}(\cdot)$ be the corresponding Weyl function. Assume also that $Y$ is an isomorphism in $\mathfrak{H} \oplus \mathfrak{H}$ given by

$$
Y\left\{f, f^{\prime}\right\}=\left\{f^{\prime}, f+\lambda_{0} f^{\prime}\right\}, \quad\left\{f, f^{\prime}\right\} \in \mathfrak{H} \oplus \mathfrak{H}
$$

Then:

(i) $S:=\left(A-\lambda_{0}\right)^{-1}$ is a bounded symmetric operator with the closed domain $\operatorname{dom} S=$ $\operatorname{ran}\left(A-\lambda_{0}\right)^{-1}$

(ii) $Y\left(S^{*}\right)=A^{*}$ and the triplet $\dot{\Pi}=\left\{\mathcal{H} \oplus \mathcal{H}, \dot{\Gamma}, \dot{\Gamma}^{\top}\right\}$ with the mappings

$$
\dot{\Gamma}=\left(\begin{array}{c}
\dot{\Gamma}_{0} \\
\dot{\Gamma}_{1}
\end{array}\right): S^{*} \rightarrow \mathcal{H} \oplus \mathcal{H} \quad \text { and } \quad \dot{\Gamma}^{\top}=\left(\begin{array}{c}
\dot{\Gamma}_{0}^{\top} \\
\dot{\Gamma}_{1}^{\top}
\end{array}\right): S^{*} \rightarrow \mathcal{H} \oplus \mathcal{H}
$$

given by

$$
\dot{\Gamma}_{0}=\Gamma_{0} Y \uparrow S^{*}, \quad \dot{\Gamma}_{1}=-\Gamma_{1} Y \uparrow S^{*}, \quad \dot{\Gamma}_{0}^{\top}=\Gamma_{0}^{\top} Y \uparrow S^{*}, \quad \dot{\Gamma}_{1}^{\top}=-\Gamma_{1}^{\top} Y \uparrow S^{*}
$$

is a boundary triplet for $\{S, S\}$ which belongs to the class $B T_{\infty}$;

(iii) $S_{0}\left(=\operatorname{ker} \dot{\Gamma}_{0}\right)=\left(A_{0}-\lambda_{0}\right)^{-1}$ and the corresponding Weyl function is

$$
M_{\dot{\Pi}}(z)=-M_{\Pi}\left(\lambda_{0}+\frac{1}{z}\right), \quad z \in \rho\left(S_{0}\right), \quad z \neq 0 .
$$

Proof. (i) The statement is implied by the inclusion $\lambda_{0} \in \widehat{\rho}(A)$.

(ii) It is clear from (4.16) that

$$
Y^{-1}\left\{g, g^{\prime}\right\}=\left\{g^{\prime}-\lambda_{0} g, g\right\}, \quad\left\{g, g^{\prime}\right\} \in \mathfrak{H} \oplus \mathfrak{H} .
$$

This together with (4.16) gives the following identities for each relation $T \in \widetilde{\mathcal{C}}(\mathfrak{H})$ :

$$
Y(T)=T^{-1}+\lambda_{0} I, \quad Y^{-1}(T)=\left(T-\lambda_{0} I\right)^{-1} .
$$

In particular, $Y\left(S^{*}\right)=S^{-1 *}+\lambda_{0} I=A^{*}$ and, therefore, the operators in (4.17) are well defined. Now, it is straightforward to check that the Green's identity (2.11) for the triplet $\Pi$ yields the same identity (2.11) for the operators defined in (4.17). Moreover, the operators $\dot{\Gamma}$ and $\dot{\Gamma}^{\top}$ are surjective, because so are the operators $\dot{\Gamma}$ and $\dot{\Gamma}^{\top}$, and $Y\left(S^{*}\right)=A^{*}$. This shows that $\dot{\Pi}$ is a boundary triplet for $\{S, S\}$.

On the other hand, from the first equality in (4.17) one obtains

$$
S_{0}:=\operatorname{ker} \dot{\Gamma}_{0}=Y^{-1}\left(A_{0}\right)=\left(A_{0}-\lambda_{0}\right)^{-1},
$$

so that $S_{0} \in[\mathfrak{H}]$. Moreover, for each $n \in \mathfrak{N}_{S}(=\mathfrak{H} \ominus \operatorname{dom} S)$ one has $Y\{0, n\}=\left\{n, \lambda_{0} n\right\}$ and hence

$$
\dot{\Gamma}_{0}\{0, n\}=\Gamma_{0}\left\{n, \lambda_{0} n\right\}=\Gamma_{0}^{\top}\left\{n, \lambda_{0} n\right\}=\dot{\Gamma}_{0}^{\top}\{0, n\} .
$$

Therefore, the triplet $\dot{\Pi}$ belongs to the class $B T_{\infty}$.

(iii) If $z \neq 0, z \in \rho(S)$ and $f \in \mathfrak{N}_{z}(S)$, then by (4.17)

$$
\begin{gathered}
\dot{\Gamma}_{0}\{f, z f\}=\Gamma_{0} Y\{f, z f\}=z \Gamma_{0}\left\{f,\left(\lambda_{0}+\frac{1}{z}\right) f\right\}, \\
\dot{\Gamma}_{1}\{f, z f\}=-z \Gamma_{1}\left\{f,\left(\lambda_{0}+\frac{1}{z}\right) f\right\} .
\end{gathered}
$$

Combining these equalities with the definition (2.12) of the Weyl function yields formula (4.18) for $M_{\dot{\Pi}}(\cdot)$.

Now we are ready to prove the main theorems in this section which improve Theorem 3.9 for the case of operators $A_{0}^{(j)}$ with a real regular point. 
Theorem 4.6. Let $\mathfrak{H}_{j}$ be a Hilbert space, let $A^{(j)} \in\left[\mathcal{D}_{j}, \mathfrak{H}_{j}\right]$ be a bounded simple symmetric operator with the closed domain $\mathcal{D}_{j} \subset \mathfrak{H}_{j}$ and let $\Pi_{j}=\left\{\mathcal{H} \oplus \mathcal{H}, \Gamma^{(j)}, \Gamma^{\top(j)}\right\} \in B T_{\infty}$ be a boundary triplet for $\left\{A^{(j)}, A^{(j)}\right\}$ with the mappings

$$
\Gamma^{(j)}=\left(\begin{array}{c}
\Gamma_{0}^{(j)} \\
\Gamma_{1}^{(j)}
\end{array}\right): A^{(j) *} \rightarrow \mathcal{H} \oplus \mathcal{H}, \quad \Gamma^{\top(j)}=\left(\begin{array}{l}
\Gamma_{0}^{\top(j)} \\
\Gamma_{1}^{\top}(j)
\end{array}\right): A^{(j) *} \rightarrow \mathcal{H} \oplus \mathcal{H} .
$$

Moreover, let $A_{0}^{(j)}\left(=\operatorname{ker} \Gamma_{0}^{(j)}\right)$ be a bounded non-self-adjoint operator and let $M_{\Pi_{j}}(\cdot)$ be the corresponding Weyl function, $j \in\{1,2\}$. If for some $R>0$,

$$
M_{\Pi_{1}}(z)=M_{\Pi_{2}}(z), \quad|z|>R,
$$

then the boundary triplets $\Pi_{1}$ and $\Pi_{2}$ are unitarily equivalent.

Proof. Let $\mathfrak{N}_{j}=\mathfrak{H}_{j} \ominus \mathcal{D}_{j}$ and let $\gamma_{\Pi_{j}} \in\left[\mathcal{H}, \mathfrak{N}_{j}\right], \gamma_{\Pi_{\top}, j} \in\left[\mathcal{H}, \mathfrak{N}_{j}\right]$ and $\mathcal{F}_{\Pi_{j}} \in[\mathcal{H}]$ be the operators in (4.4) corresponding to the triplet $\Pi_{j}, j \in\{1,2\}$. Then according to Definition $4.2 \gamma_{\Pi_{j}}=\gamma_{\Pi_{\top}, j}=: \gamma_{j}$ and hence (4.6) yields

$$
M_{\Pi_{j}}(z)=\mathcal{F}_{\Pi_{j}}+\gamma_{j}^{*}\left(A_{0}^{(j)}-z\right)^{-1} \gamma_{j}, \quad z \in \rho\left(A_{0}^{(j)}\right), \quad j \in\{1,2\} .
$$

Clearly, $\mathcal{F}_{\Pi_{j}}=\lim _{z \rightarrow \infty} M_{\Pi_{j}}(z)$ and now it follows from (4.19) that

$$
\begin{aligned}
\mathcal{F}_{\Pi_{1}} & =\mathcal{F}_{\Pi_{2}}=: \mathcal{F}, \\
\gamma_{1}^{*}\left(P_{\mathfrak{N}_{1}}\left(A_{0}^{(1)}-z\right)^{-1}\left\lceil\mathfrak{N}_{1}\right) \gamma_{1}\right. & =\gamma_{2}^{*}\left(P_{\mathfrak{N}_{2}}\left(A_{0}^{(2)}-z\right)^{-1}\left\lceil\mathfrak{N}_{2}\right) \gamma_{2}, \quad|z|>R .\right.
\end{aligned}
$$

Let $\widetilde{A}_{0}^{(j)} \in\left[\mathfrak{H}_{j}\right]$ be a self-adjoint extension of $A^{(j)}$ defined by

$$
\widetilde{A}_{0}^{(j)}=\left(\begin{array}{cc}
A_{00}^{(j)} & A_{10}^{(j) *} \\
A_{10}^{(j)} & 0
\end{array}\right): \mathcal{D}_{j} \oplus \mathfrak{N}_{j} \rightarrow \mathcal{D}_{j} \oplus \mathfrak{N}_{j}, \quad j \in\{1,2\},
$$

(i.e., by (4.2) with $B=0$ ) and define the mappings $\widetilde{\Gamma}_{0}^{(j)}, \widetilde{\Gamma}_{1}^{(j)}: A^{(j) *} \rightarrow \mathcal{H}, \quad j \in\{1,2\}$, by

$$
\widetilde{\Gamma}_{0}^{(j)}\left\{f, f^{\prime}\right\}=\gamma_{j}^{*} P_{\mathfrak{N}_{j}} f, \quad \widetilde{\Gamma}_{1}^{(j)}\left\{f, f^{\prime}\right\}=\gamma_{j}^{-1}\left(f^{\prime}-\widetilde{A}_{0}^{(j)} f\right), \quad\left\{f, f^{\prime}\right\} \in A^{(j) *} .
$$

It follows from $\left[14\right.$, Proposition 3.5] that the collection $\widetilde{\Pi}_{j}=\left\{\mathcal{H}, \widetilde{\Gamma}_{0}^{(j)}, \widetilde{\Gamma}_{1}^{(j)}\right\}$ is an ordinary boundary triplet for $A^{(j) *}$ with the Weyl function

$$
\widetilde{M}_{j}(z)=\gamma_{j}^{-1}\left(z I+A_{10}^{(j)}\left(A_{00}^{(j)}-z\right)^{-1} A_{10}^{(j) *}\right) \gamma_{j}^{-1 *}, \quad z \in \rho\left(A_{00}^{(j)}\right), \quad j \in\{1,2\} .
$$

Next, in view of (4.2) the operator $A_{0}^{(j)}$ has the block representation

$$
A_{0}^{(j)}=\left(\begin{array}{cc}
A_{00}^{(j)} & A_{10}^{(j) *} \\
A_{10}^{(j)} & B_{j}
\end{array}\right): \mathcal{D}_{j} \oplus \mathfrak{N}_{j} \rightarrow \mathcal{D}_{j} \oplus \mathfrak{N}_{j}, \quad j \in\{1,2\}
$$

with some $B_{j} \in\left[\mathfrak{N}_{j}\right]$. Applying the Frobenius formula to (4.26) and taking (4.25) into account one gets

$$
\begin{gathered}
P_{\mathfrak{N}_{j}}\left(A_{0}^{(j)}-z\right)^{-1} \uparrow \mathfrak{N}_{j}=\left(B_{j}-z I-A_{10}^{(j)}\left(A_{00}^{(j)}-z\right)^{-1} A_{10}^{(j) *}\right)^{-1} \\
=\left(B_{j}-\gamma_{j} \widetilde{M}_{j}(z) \gamma_{j}^{*}\right)^{-1}=\gamma_{j}^{-1 *}\left(\gamma_{j}^{-1} B_{j} \gamma_{j}^{-1 *}-\widetilde{M}_{j}(z)\right)^{-1} \gamma_{j}^{-1}, \quad j \in\{1,2\} .
\end{gathered}
$$

Substituting these identities into (4.22) yields the equality

$$
\widetilde{B}_{1}-\widetilde{M}_{1}(z)=\widetilde{B}_{2}-\widetilde{M}_{2}(z), \quad|z|>R,
$$


where $\widetilde{B}_{j}=\gamma_{j}^{-1} B_{j} \gamma_{j}^{-1 *}(\in[\mathcal{H}])$. It follows easily from (4.27) and (4.25) that

$$
\begin{gathered}
\widetilde{B}_{1}=\widetilde{B}_{2}=: \widetilde{B}, \\
\widetilde{M}_{1}(z)=\widetilde{M}_{2}(z), \quad z \in \mathbb{C} \backslash \mathbb{R},
\end{gathered}
$$

(cf. Corollary 3.2(ii)). On the other hand, the equality (4.8) together with (4.23) and (4.26) gives

$$
\begin{gathered}
\Gamma_{0}^{(j)}\left\{f, f^{\prime}\right\}=\gamma_{j}^{-1}\left(f^{\prime}-A_{0}^{(j)} f\right)=\gamma_{j}^{-1}\left(f^{\prime}-\widetilde{A}_{0}^{(j)} f\right)-\gamma_{j}^{-1} B_{j} P_{\mathfrak{N}_{j}} f \\
=\gamma_{j}^{-1}\left(f^{\prime}-\widetilde{A}_{0}^{(j)} f\right)-\widetilde{B}_{j} \gamma_{j}^{*} P_{\mathfrak{N}_{j}} f, \\
\Gamma_{1}^{(j)}\left\{f, f^{\prime}\right\}=-\gamma_{j}^{*} P_{\mathfrak{N}_{j}} f+\mathcal{F}_{\Pi_{j}} \gamma_{j}^{-1}\left(f^{\prime}-\widetilde{A}_{0}^{(j)} f\right)-\mathcal{F}_{\Pi_{j}} \widetilde{B}_{j} \gamma_{j}^{*} P_{\mathfrak{N}_{j}} f \\
=\left(-I_{\mathcal{H}}-\mathcal{F}_{\Pi_{j}} \widetilde{B}_{j}\right) \gamma_{j}^{*} P_{\mathfrak{N}_{j}} f+\mathcal{F}_{\Pi_{j}} \gamma_{j}^{-1}\left(f^{\prime}-\widetilde{A}_{0}^{(j)} f\right) .
\end{gathered}
$$

Comparing these equalities with (4.24) and taking (4.21) and (4.28) into account we obtain for $j \in\{1,2\}$ and all $\left\{f, f^{\prime}\right\} \in A^{(j) *}$,

$$
\begin{gathered}
\Gamma_{0}^{(j)}\left\{f, f^{\prime}\right\}=\widetilde{\Gamma}_{1}^{(j)}\left\{f, f^{\prime}\right\}-\widetilde{B}^{(j)}\left\{f, f^{\prime}\right\}, \\
\Gamma_{1}^{(j)}\left\{f, f^{\prime}\right\}=\left(-I_{\mathcal{H}}-\mathcal{F} \widetilde{B}\right) \widetilde{\Gamma}_{0}^{(j)}\left\{f, f^{\prime}\right\}+\mathcal{F} \widetilde{\Gamma}_{1}^{(j)}\left\{f, f^{\prime}\right\} .
\end{gathered}
$$

With $X=\left(\begin{array}{cc}-\widetilde{B} & I_{\mathcal{H}} \\ -I_{\mathcal{H}}-\mathcal{F} \widetilde{B} & \mathcal{F}\end{array}\right)$ and $\widetilde{\Gamma}^{(j)}=\left(\begin{array}{l}\widetilde{\Gamma}_{0}^{(j)} \\ \widetilde{\Gamma}_{1}^{(j)}\end{array}\right)$ one can rewrite the previous two equalities in the form

$$
\Gamma^{(j)}=X \widetilde{\Gamma}^{(j)}, \quad j \in\{1,2\} .
$$

It follows from (4.29) and Theorem 2.16 that the ordinary boundary triplets $\widetilde{\Pi}_{1}$ and $\widetilde{\Pi}_{2}$ are unitarily equivalent, that is

$$
\widetilde{U}\left(A^{(1) *}\right)=A^{(2) *} \text { and } \widetilde{\Gamma}^{(2)} \widetilde{U} \uparrow A^{(1) *}=\widetilde{\Gamma}^{(1)} .
$$

with some unitary operator $\widetilde{U} \in\left[\mathfrak{H}_{1}, \mathfrak{H}_{2}\right]$. This and (4.30) prove the unitary equivalence of the triplets $\Pi_{1}$ and $\Pi_{2}$.

Theorem 4.7. Let $A^{(j)}$ be a, not necessarily densely defined, simple symmetric operator in $\mathfrak{H}_{j}$, let $\lambda_{0}=\bar{\lambda}_{0} \in \widehat{\rho}\left(A^{(j)}\right)$, and let $\Pi_{j}=\left\{\mathcal{H} \oplus \mathcal{H}, \Gamma^{(j)}, \Gamma^{\top(j)}\right\} \in B T_{\lambda_{0}}$ be a boundary triplet for $\left\{A^{(j)}, A^{(j)}\right\}$ with the Weyl function $M_{\Pi_{j}}(\cdot), j \in\{1,2\}$. If for some $\varepsilon>0$,

$$
M_{\Pi_{1}}(z)=M_{\Pi_{2}}(z), \quad\left|z-\lambda_{0}\right|<\varepsilon,
$$

then the boundary triplets $\Pi_{1}$ and $\Pi_{2}$ are unitarily equivalent.

Proof. Let $S^{(j)}=\left(A^{(j)}-\lambda_{0}\right)^{-1}$, let $\dot{\Pi}_{j}=\left\{\mathcal{H} \oplus \mathcal{H}, \dot{\Gamma}^{(j)}, \dot{\Gamma}^{\top(j)}\right\} \in B T_{\infty}$ be a boundary triplet for $\left\{S^{(j)}, S^{(j)}\right\}$ constructed in Lemma 4.5 and let $M_{\dot{\Pi}_{j}}(z)=-M_{\Pi_{j}}\left(\lambda_{0}+\frac{1}{z}\right)$ be the Weyl function for $\dot{\Pi}_{j}$ (see (4.18)). Then in view of (4.31) for some $R>0$ one has

$$
M_{\dot{\Pi}_{1}}(z)=M_{\dot{\Pi}_{2}}(z), \quad|z|>R .
$$

Therefore according to Theorem 4.6 the triplets $\dot{\Pi}_{1}$ and $\dot{\Pi}_{2}$ are unitarily equivalent, which implies that the triplets $\Pi_{1}$ and $\Pi_{2}$ are unitarily equivalent as well.

Corollary 4.8. Let $\mathcal{H}, \mathfrak{H}_{1}$ and $\mathfrak{H}_{2}$ be Hilbert spaces, let $\mathfrak{N}_{j}$ be a subspace of $\mathfrak{H}_{j}$ and let $\widetilde{A}_{j} \in\left[\mathfrak{H}_{j}\right], F_{j} \in[\mathcal{H}]$ and $K_{j} \in\left[\mathcal{H}, \mathfrak{H}_{j}\right]$ be operators such that $\mathfrak{N}_{j} \supset \operatorname{ran}\left(\widetilde{A}_{j}-\right.$ $\left.\widetilde{A}_{j}^{*}\right)$, ker $K_{j}=\{0\}$ and $\operatorname{ran} K_{j}=\mathfrak{N}_{j}, j \in\{1,2\}$. Assume also that

$$
\overline{\operatorname{span}}\left\{\widetilde{A}_{1}^{n} \mathfrak{N}_{1}: n=0,1, \ldots\right\}=\mathfrak{H}_{1} \quad \text { and } \overline{\operatorname{span}}\left\{\widetilde{A}_{2}^{n} \mathfrak{N}_{2}: n=0,1, \ldots\right\}=\mathfrak{H}_{2} .
$$


If under the above assumptions the equality

$$
F_{1}+K_{1}^{*}\left(\widetilde{A}_{1}-z\right)^{-1} K_{1}=F_{2}+K_{2}^{*}\left(\widetilde{A}_{2}-z\right)^{-1} K_{2}, \quad|z|>R,
$$

holds for some $R>0$, then there exists a unitary operator $U \in\left[\mathfrak{H}_{1}, \mathfrak{H}_{2}\right]$ such that

$$
U K_{1}=K_{2} \quad \text { and } \quad U \widetilde{A}_{1}=\widetilde{A}_{2} U .
$$

Proof. Let $\mathcal{D}_{j}=\mathfrak{H}_{j} \ominus \mathfrak{N}_{j}, j \in\{1,2\}$. Since $\mathcal{D}_{j} \subset \operatorname{ker}\left(\widetilde{A}_{j}-\widetilde{A}_{j}^{*}\right)$, the operator $A^{(j)}:=\widetilde{A}_{j} \uparrow$ $\mathcal{D}_{j}$ is symmetric in $\mathfrak{H}_{j}$ with the closed domain $\mathcal{D}_{j}, j \in\{1,2\}$. Moreover, the relations (4.32) imply the simplicity of the operators $A^{(1)}$ and $A^{(2)}$.

Letting in (4.10) and (4.11) $K=K_{j}, \widetilde{A}=\widetilde{A}_{j}$ and $F=F_{j}$ we construct the boundary triplet $\Pi_{j}=\left\{\mathcal{H} \oplus \mathcal{H}, \Gamma^{(j)}, \Gamma^{\top(j)}\right\} \in B T_{\infty}$ for $\left\{A^{(j)}, A^{(j)}\right\}$ such that the corresponding Weyl function is

$$
M_{\Pi_{j}}(z)=F_{j}+K_{j}^{*}\left(\widetilde{A}_{j}-z\right)^{-1} K_{j}, \quad j \in\{1,2\}
$$

(see Proposition 4.1). Since by (4.33) $M_{\Pi_{1}}(z)=M_{\Pi_{2}}(z),|z|>R$, it follows from Theorem 4.6 that the triplets $\Pi_{1}$ and $\Pi_{2}$ are unitarily equivalent by means of a unitary operator $U \in\left[\mathfrak{H}_{1}, \mathfrak{H}_{2}\right]$. In particular, this yields the relations in (4.34).

Remark 4.9. (i) In the case that $\mathcal{H}_{j}=\mathfrak{N}_{j}, F_{j}=0$ and $K_{j}=I_{\mathfrak{N}_{j}}, j \in\{1,2\}$, the equality (4.33) takes the form

$$
P_{\mathfrak{N}_{1}}\left(\widetilde{A}_{1}-z\right)^{-1} \uparrow \mathfrak{N}_{1}=P_{\mathfrak{N}_{2}}\left(\widetilde{A}_{2}-z\right)^{-1} \uparrow \mathfrak{N}_{2}, \quad|z|>R
$$

For this case the statement of Corollary 4.8 was proved in [1, Theorem 6.2].

(ii) As it is known (see for instance [5]) a linear stationary dynamical discrete-time system (LSDS) is a collection

$$
\alpha=\left\{\widetilde{A}, K, N, F ; \mathfrak{H}, \mathcal{H}_{0}, \mathcal{H}_{1}\right\}
$$

of Hilbert spaces $\mathfrak{H}, \mathcal{H}_{0}, \mathcal{H}_{1}$, and operators $\widetilde{A} \in[\mathfrak{H}], K \in\left[\mathcal{H}_{0}, \mathfrak{H}\right], \quad N \in\left[\mathcal{H}_{1}, \mathfrak{H}\right]$ and $F \in\left[\mathcal{H}_{0}, \mathcal{H}_{1}\right]$. Moreover, the operator function

$$
\Theta_{\alpha}(z)=F+N^{*}(\widetilde{A}-z)^{-1} K, \quad z \in \rho(\widetilde{A}),
$$

is called the transfer function of the system $\alpha$.

Two systems $\alpha_{j}=\left\{\widetilde{A}_{j}, K_{j}, N_{j}, F_{j} ; \mathfrak{H}_{j}, \mathcal{H}_{0}, \mathcal{H}_{1}\right\}, j \in\{1,2\}$, are called similar (unitarily similar) if there is an operator $U \in\left[\mathfrak{H}_{1}, \mathfrak{H}_{2}\right]$ with $0 \in \rho(U)$ (resp. unitary $U$ ) such that

$$
U \widetilde{A}_{1}=\widetilde{A}_{2} U, \quad U K_{1}=K_{2}, \quad U N_{1}=N_{2} .
$$

Some sufficient conditions for similarity and unitary similarity of $L S D S$ with the same transfer function has been discovered in $[3,5]$ (for systems of other types see [4]). For systems involving normal main operators $\widetilde{A}_{j}$ some sufficient conditions for their unitary similarity have been obtained in [2].

If in addition the system (4.35) satisfies the conditions

$$
\begin{gathered}
\mathcal{H}_{0}=\mathcal{H}_{1}=: \mathcal{H}, \quad N=K, \quad 0 \in \widehat{\rho}(K), \quad \operatorname{ran} K \supset \operatorname{ran}\left(\widetilde{A}-\widetilde{A}^{*}\right), \\
\overline{\operatorname{span}}\left\{\widetilde{A}^{n} K \mathcal{H}: n=0,1, \ldots\right\}=\mathfrak{H}
\end{gathered}
$$

(the relation (4.38) means that the system $\alpha$ is simple in the sense of $[3,5]$ ), then the transfer function takes the form

$$
\Theta_{\alpha}(z)=F+K^{*}(\widetilde{A}-z)^{-1} K, \quad z \in \rho(\widetilde{A})
$$

and in view of Lemma 4.3 and Proposition $4.1 \Theta_{\alpha}(\cdot)$ is the Weyl function corresponding to some boundary triplet $\Pi \in B T_{\infty}$. Moreover, it follows from (4.39) and Corollary 4.8 that the transfer function defines the system (4.35) satisfying (4.37) and (4.38) uniquely up to unitary similarity. 
In [2] passive systems of the form (4.37) (i.e. the associated $2 \times 2$ block operator with entries $\widetilde{A}, K^{*}, K$, and $F$ is contractive) are called passive quasi-selfadjoint systems, or shortly $p q s$-systems. For such systems Corollary 4.8 was proved in another way in [2, Proposition 4.3], see also [2, Theorem 3.5] for an extension of this result where normal main operators $\widetilde{A}_{j}$ are allowed.

\section{Negative Results}

Theorem 5.1. Let $A^{(1)}$ be a simple symmetric operator in $\mathfrak{H}_{1}$, let $\Pi_{1}=\left\{\mathcal{H}, \Gamma_{0}^{(1)}, \Gamma_{1}^{(1)}\right\}$ be an ordinary boundary triplet for $A^{(1) *}$ and $M_{1}(\cdot)$ the corresponding Weyl function. If $B_{1} \in[\mathcal{H}]$ and $0 \in \rho\left(M_{1}\left(z_{0}\right)-B_{1}\right)$ for some $z_{0} \in \mathbb{C}_{+}$, then the following statements hold:

(i) There exists a (non-unique) simple symmetric operator $A^{(2)}$, an ordinary boundary triplet $\Pi_{2}=\left\{\mathcal{H}, \Gamma_{0}^{(2)}, \Gamma_{1}^{(2)}\right\}$ for $A^{(2) *}$, a (non-unique) bounded dissipative operator $B_{2}$, and an open neighborhood $\Omega_{+} \subset \mathbb{C}_{+}$of $z_{0}$, such that the following equality holds

$$
\left(B_{1}-M_{1}(z)\right)^{-1}=\left(B_{2}-M_{2}(z)\right)^{-1}, \quad z \in \Omega_{+} .
$$

(ii) The boundary triplets $\widehat{\Pi}_{I, B_{1}}$ and $\widehat{\Pi}_{I, B_{2}}$ defined in Lemma 3.8 are not unitarily equivalent.

(iii) If $B_{1}$ is not dissipative, the extensions

$$
A_{B_{1}}^{(1)}=\operatorname{ker}\left(\Gamma_{1}^{(1)}-B_{1} \Gamma_{0}^{(1)}\right) \quad \text { and } \quad A_{B_{2}}^{(2)}=\operatorname{ker}\left(\Gamma_{1}^{(2)}-B_{2} \Gamma_{0}^{(2)}\right)
$$

are not unitarily similar.

If in addition $B_{1}$ is accumulative and $B_{1} \neq B_{1}^{*}$, then $0 \in \rho\left(M_{1}\left(z_{0}\right)-B_{1}\right)$ for every $z_{0} \in \mathbb{C}_{+}$and the statement $(i)$ holds true with $\Omega_{+}=\mathbb{C}_{+}$. In particular, the extensions $A_{B_{1}}^{(1)}$ and $A_{B_{2}}^{(2)}$ are not unitarily similar.

Proof. (i) Since $M_{1}(\cdot)$ belongs to $R[\mathcal{H}]$ the assumption $0 \in \rho\left(M_{1}\left(z_{0}\right)-B_{1}\right)$ implies that there exists an open neighborhood $\Omega_{+} \subset \mathbb{C}_{+}$of $z_{0}$, such that $0 \in \rho\left(M_{1}(z)-B_{1}\right)$ for all $z \in \Omega_{+}$. Now choose a dissipative operator $B \in[\mathcal{H}]$ such that $\operatorname{Re} B \neq 0$, and the sum $B_{2}:=B+B_{1}$ is also dissipative and define the function $M_{2}(\cdot)$ by

$$
M_{2}(z):=M_{1}(z)+B, \quad z \in \mathbb{C}_{+} ; \quad M_{2}(z):=M_{1}(z)+B^{*}, \quad z \in \mathbb{C}_{-} .
$$

Since $M_{1}(\cdot)$ is the Weyl function corresponding to the ordinary boundary triplet $\Pi_{1}$, $M_{1}(\cdot) \in R^{u}[\mathcal{H}]$, i.e. $M_{1}(\cdot) \in R[\mathcal{H}]$ and $0 \in \rho(\operatorname{Im} M(i))$. Combining this fact with the inequality $\operatorname{Im} B \geq 0$ we get that $M_{2}(\cdot) \in R^{u}[\mathcal{H}]$. Therefore (see [14]) there exists a simple symmetric operator $A^{(2)}$ and an ordinary boundary triplet $\Pi_{2}=\left\{\mathcal{H}, \Gamma_{0}^{(2)}, \Gamma_{1}^{(2)}\right\}$ for $A^{(2) *}$ such that the corresponding Weyl function is equal to $M_{2}(\cdot)$. By construction, $B=B_{2}-B_{1} \neq 0$ is dissipative and (5.3) implies that

$$
M_{2}(z)-B_{2}=M_{1}(z)-B_{1}, \quad z \in \mathbb{C}_{+} .
$$

Since $0 \in \rho\left(M_{1}(z)-B_{1}\right)$ for $z \in \Omega_{+}$, the last identity leads to the inclusion $0 \in \rho\left(M_{2}(z)-\right.$ $B_{2}$ ) for $z \in \Omega_{+}$. Taking inverses of both sides of (5.4) yields (5.1).

(ii) By Lemma 3.8, a collection $\widehat{\Pi}_{I, B_{j}}=\left\{\mathcal{H}, \widehat{\Gamma}_{0}^{(j)}, \widehat{\Gamma}_{1}^{(j)}\right\}, j \in\{1,2\}$, with

$$
\widehat{\Gamma}_{0}^{(j)}=\Gamma_{0}^{(j)}, \quad \widehat{\Gamma}_{1}^{(j)}=\left(\Gamma_{1}^{(j)}-\left(\operatorname{Re} B_{j}\right) \Gamma_{0}^{(j)}\right), \quad j \in\{1,2\},
$$

defines an ordinary boundary triplet for $A^{(j) *}$ such that the corresponding Weyl function is

$$
\widehat{M}_{j}(z)=M_{j}(z)-\operatorname{Re} B_{j}, \quad z \in \mathbb{C}_{+}, \quad j \in\{1,2\}
$$


According to our choice $\operatorname{Re} B \neq 0$, i.e. Re $B_{1} \neq \operatorname{Re} B_{2}$. Thus, by $(5.6) \widehat{M}_{1}(\cdot) \neq \widehat{M}_{2}(\cdot)$. By Theorem 2.16, the boundary triplets $\widehat{\Pi}_{I, B_{1}}$ and $\widehat{\Pi}_{I, B_{2}}$ are not unitarily equivalent.

Note also that as it is clear from $(5.4), M_{1}(\cdot) \neq M_{2}(\cdot)$. Therefore, by Theorem 2.16, the boundary triplets $\Pi_{1}$ and $\Pi_{2}$ are not unitarily equivalent too.

(iii) Since $\Pi_{1}$ and $\Pi_{2}$ are ordinary boundary triplets, Proposition 2.5(ii) shows that the linear relation $A_{B_{1}}^{(1)}$ is not dissipative, if so is $B_{1}$. At the same time, by Proposition 2.5(ii), $A_{B_{2}}^{(2)}$ is $m$-dissipative since, by construction, $B_{2}$ is dissipative. In particular, $A_{B_{1}}^{(1)}$ and $A_{B_{2}}^{(2)}$ are not unitarily equivalent.

Remark 5.2. (i) Theorem 5.1, as well as its proof, remains valid for generalized boundary triplets in the sense of [14]; their unitary equivalence is defined precisely in the same way as was done for ordinary boundary triplets in Definition 2.14 .

(ii) To demonstrate that Theorem 5.1 holds for a wide class of non-accumulative operators $B_{1}$, we fix $z_{0} \in \mathbb{C}_{+}$and recall that as a Weyl function of an ordinary boundary triplet $M_{1}(\cdot) \in R^{u}[\mathcal{H}]$. The latter means that $\operatorname{Im}\left(M_{1}\left(z_{0}\right) f, f\right) \geq \varepsilon_{0}\|f\|^{2}$ for some $\varepsilon_{0}>0$. Choose an arbitrary operator $B_{1} \in[\mathcal{H}]$ (not necessarily accumulative) which satisfies $\left\|\operatorname{Im} B_{1}\right\| \leq \varepsilon_{0} / 2$. Then, clearly

$$
\operatorname{Im}\left(\left(M_{1}\left(z_{0}\right)-B_{1}\right) f, f\right) \geq \operatorname{Im}\left(M_{1}\left(z_{0}\right) f, f\right)-\left|\operatorname{Im}\left(B_{1} f, f\right)\right| \geq \frac{\varepsilon_{0}}{2}\|f\|^{2} .
$$

This implies that $0 \in \rho\left(M_{1}\left(z_{0}\right)-B_{1}\right)$ and hence there exists an open neighborhood $\Omega_{+} \subset \mathbb{C}_{+}$of $z_{0}$, such that $0 \in \rho\left(M_{1}(z)-B_{1}\right)$ for all $z \in \Omega_{+}$. Again the operator $A_{B_{1}}$ is not dissipative, if $B_{1}$ is not dissipative, and thus all the conclusions of Theorem 5.1 hold.

(iii) Let under the assumptions of Theorem $5.1 n_{ \pm}\left(A^{(1)}\right)=n<\infty$, so that $\operatorname{dim} \mathcal{H}=n$. Then for any fixed $z_{0} \in \mathbb{C}_{+}$the inequality $\operatorname{det}\left(M_{1}\left(z_{0}\right)-B_{1}\right) \neq 0$ holds for almost every (with respect to the Lebesgue measure in $\mathbb{C}^{n^{2}}$ ) non-dissipative matrices $B_{1} \in \mathbb{C}^{n \times n}$. Therefore in this case the conclusions of Theorem 5.1 are valid for almost every non-dissipative matrix $B_{1} \in \mathbb{C}^{n \times n}$.

(iv) Reasonings of Theorem 5.1 can easily be extended to establish the following statement:

There exist simple symmetric operators $A^{(j)}$, ordinary boundary triplets $\Pi_{j}=$ $\left\{\mathcal{H}, \Gamma_{0}^{(j)}, \Gamma_{1}^{(j)}\right\}$ for $A^{(j) *}$ with the corresponding Weyl functions $M_{j}(\cdot), j \in\{1,2\}$, a selfadjoint operator $B_{1} \in[\mathcal{H}]$ and an accumulative operator $B_{2} \in[\mathcal{H}]$, such that

$$
\widetilde{M}_{1}(z):=\left(B_{1}-M_{1}(z)\right)^{-1}=\left(B_{2}-M_{2}(z)\right)^{-1}=: \widetilde{M}_{2}(z), \quad z \in \mathbb{C}_{+},
$$

but the extensions (5.2) are not unitarily similar.

This statement shows that even in the case of maximal accumulative extension $\widetilde{A}_{B}$ the Weyl function $\widetilde{M}(\cdot)$ of the form (3.36) does not determine the extension uniquely up to the unitary similarity.

Example. Let $\mathcal{H}$ be a separable Hilbert space. Consider in $L^{2}(\mathbb{R}, \mathcal{H})$ the momentum operator $A_{0}=-i \frac{d}{d x}, \operatorname{dom}\left(A_{0}\right)=W^{1,2}(\mathbb{R})$ and its restriction

$$
A=-i \frac{d}{d x}, \quad \operatorname{dom}(A)=W_{0}^{1,2}\left(\mathbb{R}_{-}\right) \oplus W_{0}^{1,2}\left(\mathbb{R}_{+}\right)=\left\{f \in W^{1,2}(\mathbb{R}): f(0)=0\right\} .
$$

Clearly, $A$ is symmetric operator with equal deficiency indices $n_{ \pm}=\operatorname{dim} \mathcal{H}$.

Define the boundary triplets $\Pi_{j}=\left\{\mathcal{H}, \Gamma_{0}^{(j)}, \Gamma_{1}^{(j)}\right\}, j \in\{1,2\}$, for $A^{*}$, by setting

$$
\sqrt{2} \Gamma_{0}^{(1)} f:=[f(+0)-f(-0)], \quad \sqrt{2} \Gamma_{1}^{(1)} f:=i[f(+0)+f(-0)],
$$


and

$$
\Gamma_{0}^{(2)}:=3^{-1 / 2} \Gamma_{0}^{(1)}, \quad \Gamma_{1}^{(2)}:=\sqrt{3} \Gamma_{1}^{(1)} .
$$

It easily follows from (5.7), (5.8) that the corresponding Weyl functions $M_{j}(\cdot), j \in\{1,2\}$, are

$$
M_{1}(z)=\left\{\begin{array}{ll}
i I_{\mathcal{H}}, & z \in \mathbb{C}_{+}, \\
-i I_{\mathcal{H}}, & z \in \mathbb{C}_{-},
\end{array} \quad M_{2}(z)= \begin{cases}3 i I_{\mathcal{H}}, & z \in \mathbb{C}_{+}, \\
-3 i I_{\mathcal{H}}, & z \in \mathbb{C}_{-} .\end{cases}\right.
$$

Now, let $B_{1}=-i I_{\mathcal{H}}$ and $B_{2}=i I_{\mathcal{H}}$ and define the proper extensions $A_{B_{j}}$ of $A$ by

$$
A_{B_{j}}=A^{*}\left[\operatorname{dom} A_{B_{j}}, \quad \operatorname{dom} A_{B_{j}}=\operatorname{ker}\left(\Gamma_{1}^{(j)}-B_{j} \Gamma_{0}^{(j)}\right), \quad j \in\{1,2\} .\right.
$$

Then by Proposition $2.5 A_{B_{1}}$ is m-accumulative and $A_{B_{2}}$ is $m$-dissipative, because so are $B_{1}$ and $B_{2}$. More precisely, by rewriting $\operatorname{dom} A_{B_{1}}$ in (5.10) in the form $\operatorname{dom} A_{B_{1}}=$ $W^{1,2}\left(\mathbb{R}_{-}\right) \oplus W_{0}^{1,2}\left(\mathbb{R}_{+}\right)$, one obtains

$$
\operatorname{ker}\left(A_{B_{1}}-z\right)=\left\{e^{i z x} \chi_{-}(x) h: h \in \mathcal{H}\right\}, \quad z \in \mathbb{C}_{-}, \quad \sigma_{p}\left(A_{B_{1}}\right)=\mathbb{C}_{-}
$$

and $\sigma\left(A_{B_{1}}\right)=\overline{\mathbb{C}}_{-}$, where $\chi_{-}(\cdot)$ is the indicator function of $\mathbb{R}_{-}$. Similarly, it follows from (5.10) that

$$
\operatorname{dom} A_{B_{2}}=\left\{f \in W^{1,2}\left(\mathbb{R}_{-}\right) \oplus W^{1,2}\left(\mathbb{R}_{+}\right): \quad f(+0)=-2 f(-0)\right\} .
$$

Hence the functions

$$
f_{\lambda}(x)= \begin{cases}e^{i \lambda x}, & x>0 \\ -2^{-1} e^{i \lambda x}, & x<0\end{cases}
$$

form the complete family of (generalized) eigenfunctions of the continuous spectrum of $A_{B_{2}}$, and $\sigma\left(A_{B_{2}}\right)=\sigma_{c}\left(A_{B_{2}}\right)=\mathbb{R}$.

Thus, the operators $A_{B_{1}}$ and $A_{B_{2}}$ are not similar. At the same time,

$$
\left(B_{1}-M_{1}(z)\right)^{-1}=-i / 2 \cdot I_{\mathcal{H}}=\left(B_{2}-M_{2}(z)\right)^{-1}, \quad z \in \mathbb{C}_{+} .
$$

To prove the similarity of $A_{B_{2}}$ to $A_{0}$ consider the characteristic function $W_{2}(\cdot)$ of the operator $A_{B_{2}}$. Setting $K^{*}=K=I_{\mathcal{H}}=J$ one has $\operatorname{Im} B_{2}=I_{\mathcal{H}}=K J K^{*}$. Hence, using (5.9) one obtains

$$
\begin{aligned}
W_{2}(z)= & I_{\mathcal{H}}+2 i K^{*}\left(B_{2}^{*}-M_{2}(z)\right)^{-1} K J=I_{\mathcal{H}}+2 i\left(B_{2}^{*}-M_{2}(z)\right)^{-1} \\
& =I_{\mathcal{H}}+2 i\left(-i I_{\mathcal{H}}-3 i I_{\mathcal{H}}\right)^{-1}=1 / 2 \cdot I_{\mathcal{H}}, \quad z \in \mathbb{C}_{+} .
\end{aligned}
$$

Since $W_{2}^{-1}(z)=2 I_{\mathcal{H}}$ is bounded in $\mathbb{C}_{+}$, the Nagy-Foias theorem ([30, Theorem 9.1.2]) yields the similarity of $A_{B_{2}}$ to a self-adjoint operator.

Furthermore, it is easily seen that the operator $A_{B_{2}}$ is completely non-self-adjoint. In addition,

$$
W_{2}(x+i 0)=s-\lim _{y \downarrow 0} W_{2}(x+i y)=1 / 2 \cdot I_{\mathcal{H}}, \quad x \in \mathbb{R} .
$$

Thus, $\left\|W_{2}(x+i 0)\right\|<1$ for $x \in \mathbb{R}$ and, by [30, Corollary 9.1.3], the operator $A_{B_{2}}$ is similar to the multiplication operator $Q: f(x) \rightarrow x f(x)$ in $L^{2}(\mathbb{R}, \mathcal{H})$. It remains to note that the operator $A_{0}$ is unitarily equivalent to the multiplication operator $Q$, too.

Remark 5.3. (i) In this example $A^{(1)}=A^{(2)}=A$ and $A_{0}^{(1)}=A_{0}^{(2)}=A_{0}$. It is easily seen that the spectral measure $E_{A_{0}}(\cdot)$ of $A_{0}$ is spectrally equivalent to the Lebesgue measure $I_{\mathcal{H}} d t$. This example shows that Theorem 3.9 is sharp and the assumptions on the spectral measures $E_{A_{0}^{(1)}}$ and $E_{A_{0}^{(2)}}$ cannot be dropped.

(ii) It follows from $(5.9)$ that $\operatorname{ker}\left(B_{1}-M_{1}(z)\right)=\operatorname{ker}\left(-i I_{\mathcal{H}}+i I_{\mathcal{H}}\right)=\mathcal{H}, z \in \mathbb{C}_{-}$, and $0 \in \rho\left(B_{2}-M_{2}(z)\right), z \in \mathbb{C}_{ \pm}$. Thus, by Proposition $2.8 \sigma_{p}\left(A_{B_{1}}\right)=\mathbb{C}_{-}$and $\sigma\left(A_{B_{2}}\right) \subset \mathbb{R}$. 


\section{REFERENCES}

[1] Yu. M. Arlinskii, S. Hassi, H. de Snoo, Q-functions of quasi-self-adjoint contractions, Oper. Theory Adv. Apl. 163 (2005), 23-54.

[2] Yu. Arlinskiı̌, S. Hassi, H.S.V. de Snoo, Passive systems with a normal main operator and quasiselfadjoint systems, Complex Analysis and Operator Theory 3 (2009), no.1, 19-56.

[3] D.Z. Arov, Passive linear stationary dynamical systems, Sib. Mat. Zhurn. 20 (1979), no.2, $211-$ 228.

[4] D.Z. Arov, M.A. Nudelman, 'Passive linear stationary dynamical scattering systems with continuous time, Integr. Equat. Oper. Th. 24 (1996), 1-45.

[5] D.Z. Arov, M.A. Nudelman, Tests for the similarity of all minimal passive realizations of a fixed transfer function (scattering or resistance matrix), Mat. Sb. 193 (2002), no. 6, 3-24.

[6] J.F. Brasche, M.M. Malamud, H. Neidhart, Weyl function and spectral properties of self-adjoint extensions, Integral Equations Operator Theory 43 (2002), 264-289.

[7] M.Sh.Birman, M.Z. Solomyak, Spectral theory of self-adjoint operators in Hilbert space, Leningrad. Univ., Lenungrad, 1980; English translation: Math. Appl.(Soviet ser.), Reidel, Dordrecht, 1987.

[8] M.S. Brodskii, Triangular and Jordan representations of linear operators, Translational Mathematical Monographs, 32, AMS, Providence, RI, 1971.

[9] B. M. Brown, G.Grubb, I. G.Wood, M-functions for closed extensions of adjoint pairs of operators with applications to elliptic boundary problems, Math. Nachr. 282 (2009), no.3, 314-347.

[10] M. Brown, J. Hinchcliffe, M. Marletta, S. Naboko, I. Wood, The Abstract Titchmarsh-Weyl $M$-function for Adjoint Operator Pairs and its Relation to the Spectrum, Integral Equations Operator Theory 63 (2009), 297-320.

[11] M. Brown, M. Marletta, S. Naboko, I. Wood, Boundary triplets and M-functions for non-selfadjoint operators, with applications to elliptic PDEs and block operator matrices, J. London Math. Soc. 77 (2008), no.2, 700-718.

[12] V.A. Derkach, M.M. Malamud, Generalized resolvents and the boundary value problems for Hermitian operators with gaps, J. Funct. Anal. 95 (1991), 1-95.

[13] V.A. Derkach, M.M. Malamud, Characteristic functions of almost solvable extensions of Hermitian operators, Ukr. Mat. Zh. 44 (1992), no 4, 435-459.

[14] V.A. Derkach, M.M. Malamud, The extension theory of Hermitian operators and the moment problem, J. Math. Sciences, 73 (1995), no 2, 141-242.

[15] V. I. Gorbachuk, M. L. Gorbachuk. Boundary value problems for operator differential equations, volume 48 of Mathematics and its Applications (Soviet Series). Kluwer Academic Publishers Group, Dordrecht, 1991.

[16] G. Grubb, A characterization of the non local boundary value problems associated with an elliptic operator, Ann. Scuola Normale Superiore de Pisa 22 (1968), no 3, 425-513.

[17] G. Grubb, Extension theory for elliptyic partial differential operators with pseudodifferential methods, arXiv:1008.1081v1 [math.AP] 5 Aug 2010.

[18] S. Hassi, M. M. Malamud, V. I. Mogilevskii, Generalized resolvents and boundary triplets for dual pairs of linear relations, Methods Funct. Anal. Topology 11 (2005), no 2, 170-187.

[19] S. Hassi, M. M. Malamud, V. I. Mogilevskii, On the unitary equivalence of the proper extensions of a Hermitian operator and the Weyl function, Math. Notes 91 (2012), no 2, 302-307.

[20] M.G. Krein, H. Langer, Uber die Q-Function eines $\Pi$-hermiteschen operators in raume $\Pi_{\kappa}$, Acta Sci. Math. (Szeged), 34 (1973), 191-230.

[21] H. Langer, B. Textorious, On generalized resolvents and Q-functions of symmetric linear relations (subspaces) in Hilbert space, Pacif. J. Math. 72 (1977), no 1, 135-165.

[22] V.B. Lidskii, A non-self-ajoint operator of Sturm-Liouville type with discrete spectrum, Trans. Mosc. Math. Soc. 9 (1960), 45-79.

[23] V.E. Lyantse, O.G. Storozh, Methods of the theory of unbounded operators, Naukova Dumka, Kiev, 1983.

[24] M.M. Malamud, On the formula of generalized resolvents of a nondensely defined Hermitian operator, Ukr. Math. Zh. 44 (1992), no. 12, 1658-1688.

[25] M.M. Malamud, Spectral theory of elliptic operators in exterior domains, Russ. J. Math. Phys. 17 (2010), no 1, 96-125.

[26] M.M. Malamud, S.M. Malamud, Spectral theory of operator measures in Hilbert space, St. Petersburg Math. Journal 15 (2003), no. 3, 323-373.

[27] M.M. Malamud, V.I. Mogilevskii, On extensions of dual pairs of operators, Dop. Akad. Nauk Ukr. (1997), no.1, 30-37. 
[28] M.M. Malamud, V.I. Mogilevskii, On Weyl functions and Q-functions of dual pairs of linear relations, Dop. Akad. Nauk Ukr. (1999), no.4, 32-37.

[29] M. M. Malamud, V. I. Mogilevskii, Krein type formula for canonical resolvents of dual pairs of linear relations, Methods of Funct. Anal. and Topology 8 (2002), no.4, 72-100.

[30] B. S.-Nagy, C. Foias, Harmonic analysis of operators on Hilbert space, North-Holland, New York, 1970.

[31] M. L. Višik, On general boundary problems for elliptic differential equations, Trudy Moskov. Mat. Obsc. 1 (1952), 187-246 (Russian); Engl. transl. in Amer. Math. Soc. Transl. (2), 24 (1963), 107-172.

Department of Mathematics and Statistics, University of VaAsa, P.O. Box 700, 65101 VAASA, FinLAND

E-mail address: sha@uwasa.fi

Institute of Applied Mathematics and Mechanics, NAS of Ukraine, R. Luxemburg Str. 74, 83050 Donetsk, UKRAINE

E-mail address: mmm@telenet.dn.ua

Department of Mathematical Analysis, Lugans'K National University, Oboronna Str. 2, 91011 Lugans'K, UkRaine

E-mail address: vim@mail.dsip.net 